\title{
Estacionariedad, rompimientos y tendencias de la migración internacional de México: causas y consecuencias
}

\author{
Zeus Salvador Hernández Veleros \\ Tomás Serrano Avilés
}

Universidad Autónoma del Estado de Hidalgo, México

\section{Resumen}

El artículo analiza el proceso generador de datos de las series temporales migratorias internacionales de México por sexo para el periodo 1960-2010, bajo la consideración de estacionariedad con rompimientos y la búsqueda de una relación de largo plazo entre los flujos netos femenino y masculino; para determinar las fechas de cambio, los intervalos de las mismas y los posibles sucesos asociados con tales cambios. Son aplicadas pruebas de estacionariedad panel con múltiples rompimientos y modelos ARDL; además, son planteadas algunas de sus consecuencias, así como pronósticos de los diferente flujos migratorios, bajo la consideración de no presencia de nuevos rompimientos, otros se basan en dinámicas donde habrán cambios de tendencias, en tanto otros pronósticos tienen sustento en datos de acervos demográficos, crecimiento económico, situaciones de control migratorio e inseguridad.

Palabras clave: Migración, estacionariedad, rompimientos, ARDL.

\section{Abstract}

Stationarity, breaks and trends of the international migration of Mexico: causes and consequences

In the paper we analyze the data generation process of the time series of international migrations for Mexico by sex during the period 1960-2010, under the consideration of stationarity with breaks and the definition of a long term relationship between female net migration and male net migration; with the aim to define the break date, the confidence intervals of them, the possible facts related with the breaks. We employ stationarity panel test and a ARDL model, and else some consequences of these facts are mentioned; we forecast the different international flows under the assumption of no new breaks, other forecasts considers a dynamics with changes in trends, other projections have a root in demographic stocks, economic growth, inmigration enforcement and insecurity problems.

Key words: Migration, stationarity, breaks, ARDL. 


\section{INTRODUCCIÓN}

$\mathbf{E}$ 1 objetivo de este artículo es determinar si las series de migración internacional de México por sexo del periodo 1960-2010 presentan cambios significativos en su dinámica, los cuales se puedan interpretar como rompimientos, y en tal caso, evaluar si ello afecta a la estacionariedad de las series consideradas; asimismo, serán definidas algunas de las situaciones particulares ocurridas dentro de los intervalos de confianza de las fechas de los rompimientos como sus posibles causas o bien las consecuencias de estos cambios sobre la migración internacional y otros flujos migratorios; por último, serán expuestos tres escenarios de tendencias de la migración internacional.

En la primera sección de este artículo son retomados diversos estudios sobre los flujos migratorios internacionales de México y es presentado un análisis propio sobre la migración internacional de este país y su dinámica cambiante observada en las décadas más recientes, basados en registros o estimaciones de instituciones como el Instituto Nacional de Estadística y Geografía (INEGI), el Consejo Nacional de Población (CONAPO), la Sociedad Mexicana de Demografía (SOMEDE), el Instituto Nacional de Migración (INM), o estadounidenses como Inmigración y Control de Aduanas (ICE, por las siglas en inglés de Inmigration and Customs Enforcement).

En el apartado segundo, dividido en tres subsecciones, primero se muestran los aspectos referentes a la estacionariedad de las series temporales y cómo aquélla puede ser afectada por la presencia de rompimientos; en segundo lugar son mencionadas las particularidades de la prueba econométrica panel de estacionariedad hasta con ocho rompimientos a aplicar, la cual define los estadísticos críticos con rompimientos y dependencia entre las series mediante el uso de técnicas bootstrap. En la tercera subsección, es presentada la técnica econométrica de modelos autorregresivos y de rezagos distribuidos, ARDL, la cual puede ser empleada cuando se tienen dudas sobre si las series son estacionarias o no y se pretende evaluar la hipótesis nula de una relación de largo plazo entre las mismas.

A continuación, son presentados los datos a emplear en la investigación econométrica y cómo fueron obtenidos.

La cuarta y penúltima división confirma los resultados de la prueba de estacionariedad panel con rompimientos múltiples y del modelo ARDL, así como cuatro proyecciones: una lineal, basada en la prueba de estacio- 
nariedad; otra dinámica basado en el modelo ARDL; otra más definida a partir de la relación entre migración neta total y repatriados, y entre emigración total y repatriados, estos registrados por el Instituto Nacional de Migración (INM) y, por último, una definida mediante relaciones históricas, de acervos y flujos demográficos, de políticas de control de inmigración e inseguridad enfrentada por los migrantes entre México y Estados Unidos de América.

Cerramos con una serie de conclusiones en torno a los resultados obtenidos.

\section{Estudios SObre la Migración INTERnacional de MéXICO}

El patrón migratorio hacia Estados Unidos es un proceso social que abarca más de dos siglos, caracterizado por un constante cambio. De manera muy breve resumiremos estos avatares. Desde 1930 Gamio escribía que los mexicanos se insertaban en espacios donde no era fácil conseguir empleo, en esos años 35 por ciento de la mano de obra de la industria siderúrgica de la ciudad de Chicago provenía de México (Calavitia, 1989).

La entrada de Estados Unidos a la Segunda Guerra Mundial obligó a ese país a establecer un convenio bilateral (el Programa Bracero 19421964) para administrar la corriente de trabajadores mexicanos al sector agrícola. Aunque en un principio se pensó para abastecer mano de obra a la actividad agrícola, más adelante fue ampliado a otros sectores como el minero y el ferroviario. El Programa Bracero terminó en 1964. A lo largo de 22 años habían sido contratados poco más de cuatro millones y medios de braceros y los ilegales reportados fueron poco menos de cinco millones.

La terminación del Programa Bracero no influyó en el requerimiento de mano de obra por parte de los empleadores estadounidense, a partir de esa época comienzan los desplazamientos de mexicanos a buscar trabajo en los mercados urbanos. López (1986) señala que buscar refugio en la ciudades sobre todos para los trabajadores mexicanos sin documentos, se debió, a que en las ciudades tenían más probabilidades para ocultarse y pasar desapercibidos y, por otra, de tener un empleo usualmente con un mayor pago. Según Bustamante (1986) en este periodo la emigración de mexicanos hacia Estados Unidos consistía en un flujo circular, realizado por hombres de origen rural con escasa preparación escolar, quienes sólo permanencia algunos meses (normalmente menos de seis meses) y luego retornaban a sus comunidades de origen. El origen de estos migrantes se localizaba en los estados de Michoacán, Jalisco, Guanajuato y Zacatecas. 
Por su parte, Figueroa-Hernández y Pérez-Soto (2011) destacan que a partir de la década de los años setenta se requirió de un volumen ascendente de mano de obra, más diversificado con respecto a su perfil tradicional y factible de integrarse en los sectores de actividad económica en expansión por todo el territorio de Estados Unidos: servicios y manufactura. De este modo, a partir de finales de los años setenta e inicios de los ochenta se observó un cambio ocupacional de los migrantes mexicanos desde actividades agrícolas y estacionales hacia otras de carácter urbano, menos sujetas a variaciones de demanda y, por ende, más permanentes.

Fue en la década de los ochenta cuando se presenta la mayor legalización de mexicanos en Estados Unidos según Massey et al. (2009): más de dos millones de mexicanos fueron beneficiados con la Ley de Reforma de Inmigración y Control (IRCA por las siglas en inglés de Inmigration Reform and Control Act) de 1986, y de los procesos de reunificación familiar derivados de la regularización y posterior naturalización de muchos mexicanos, quienes podían traer legalmente a sus hijos, esposas y parientes cercanos, estos procesos provocaron una consolidación de las redes de apoyo para futuros migrantes impulsando la masificación del flujo en los años noventa; en esta década los migrantes mexicanos tienden a permanecer más tiempo en Estados Unidos, mientras nuevas entidades federativas de México se incorporan al fenómeno migratorio.

De acuerdo con Massey et al. (2009) es a partir de IRCA que empieza el proceso de militarización de la frontera, el cual se incrementa año con año; pero el control fronterizo crece dramáticamente después del ataque terrorista de 2001, y aumentan las deportaciones y la persecución al interior del país. Con la crisis económica de 2007 en Estados Unidos la migración comienza una nueva tendencia, es el final de la migración masiva hacia ese país.

Las cifras obtenidas por instituciones en estas dos naciones muestran esta tendencia, por el lado mexicano las tasas de migración internacional anualizados calculados con base en las estimaciones de ENOE, indican que para el periodo 2008 a 2014, la tasa de emigración internacional disminuyó 45.5 por ciento su nivel, al pasar de 64.1 a 34.9 por cada 10 mil habitantes. Para la inmigración internacional, la tasa anual mantiene un descenso constante desde 2008, en ese año la tasa fue de 43.6 inmigrantes por cada 10 mil habitantes; en tanto que para 2014 el indicador fue de 14.1; esto es, un descenso acumulado de 67.6 por ciento desde aquel año; por ende, el saldo neto migratorio en 2008 es muy parecido al de 2014: -20.4 y -20.8 personas por cada 10 mil, respectivamente (INEGI, 2016). 
Por su parte, el Instituto Nacional de Migración (INM, 2016) en sus cifras históricas de repatriaciones (eventos, no personas) del periodo 19962015 presenta un mínimo histórico para este último año: sólo 207 398, comparadas con el máximo histórico en el año 2000 con 1150906 repatriaciones, o con el dato del primer año de periodo señalado: 853365 devoluciones (Figura 1).

Figura 1: Repatriación de mexicanos desde Estados Unidos, 1995-2014 (eventos)

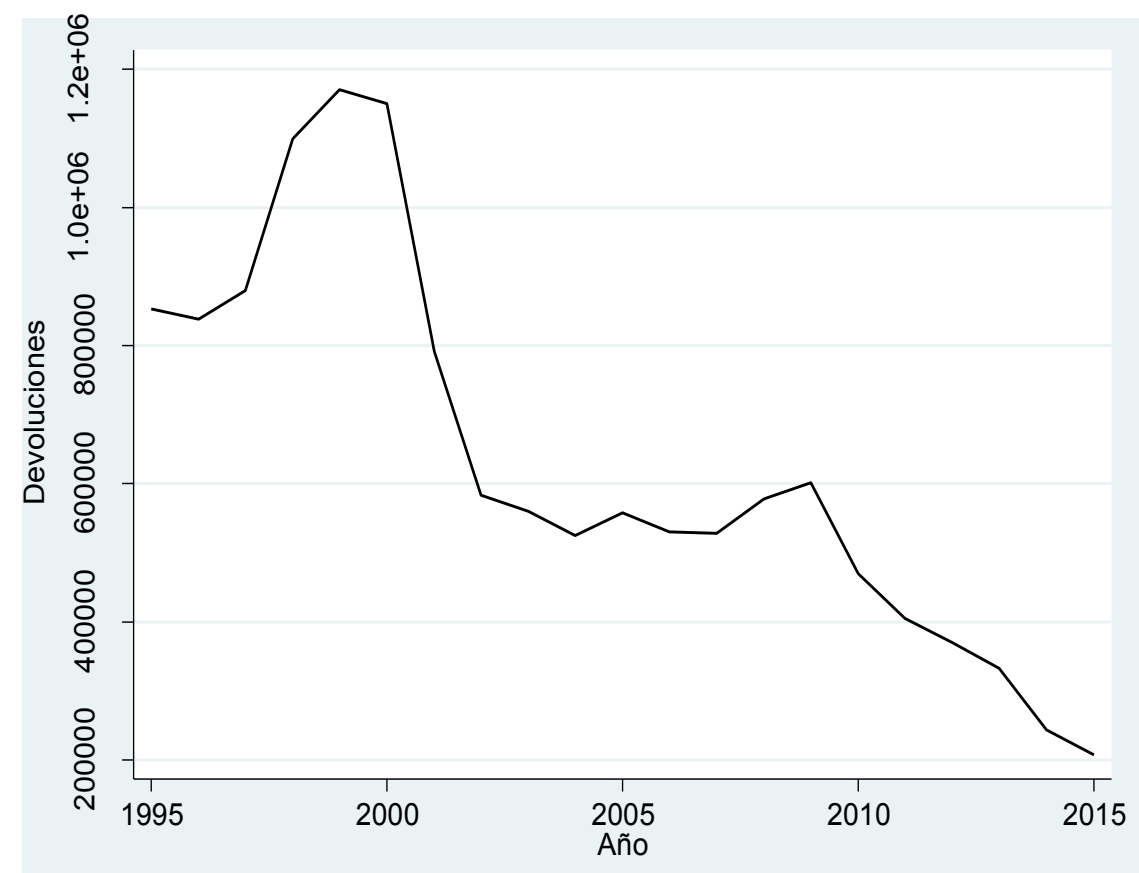

Fuente: Instituto Nacional de Migración.

Del lado estadounidense las cifras muestran que los arrestos de mexicanos en Estados Unidos en 2015 sólo representan 23.4 por ciento de los arrestos de 2004 (Figura 2); esta disminución es un claro indicador: cada vez son menos los mexicanos quienes intentan cruzar la frontera sin documentos o quienes son capturados en Estados Unidos y regresados a México. Por lo tanto, para muchos mexicanos trasladarse hacia el país colindante en el norte ha dejado de ser una opción. 
Figura 2: Mexicanos aprendidos en Estados Unidos, 2004-2015 (personas)

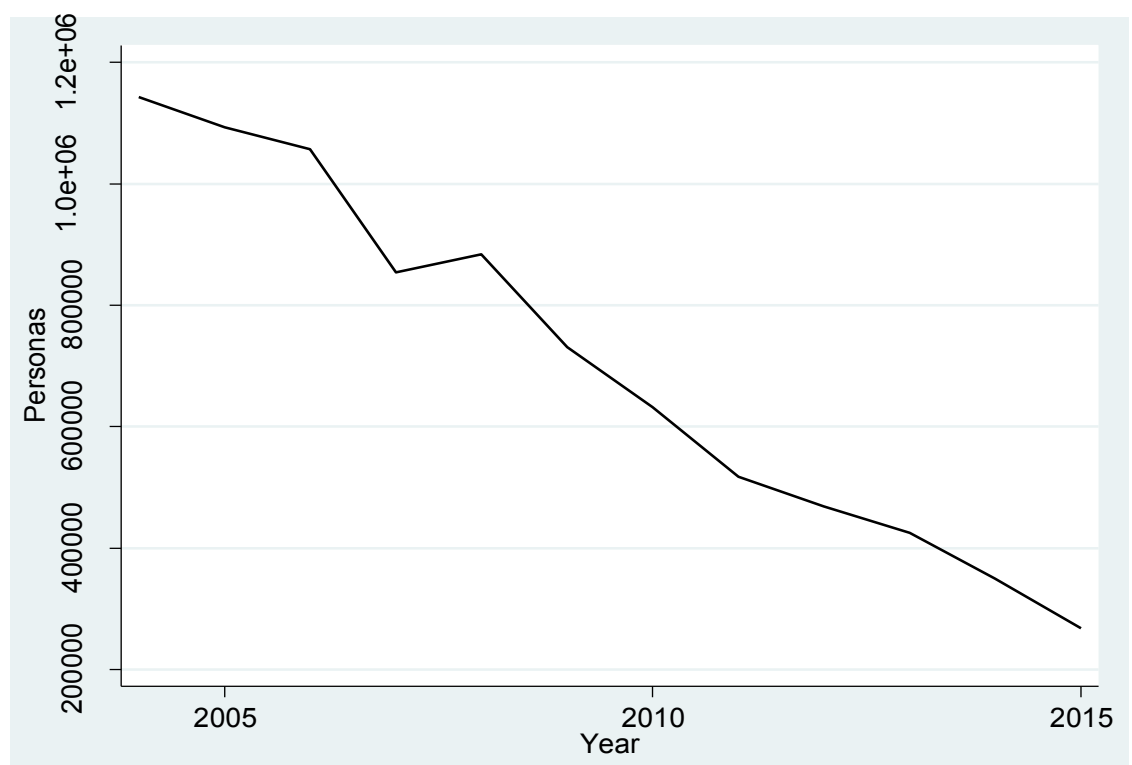

Fuente: Source: U.S. Department of Homeland Security, Customs and Border Protection (CBP) U.S. Border Patrol (USBP), Immigration and Customs Enforcement (ICE) Homeland Security Investigations (HSI), and the Office of Enforcement and Removal Operations (ERO).

\section{TÉCNICAS ECONOMÉTRICAS PARA DETERMINAR ESTACIONARIEDAD, ROMPIMIENTOS Y RELACIONES DE LARGO PLAZO}

\section{Pruebas de estacionariedad o de raíz unitaria con rompimientos}

Para exponer los aspectos teóricos de estacionariedad y rompimientos nos basaremos en todo este apartado en la obra de Hansen (2001). Este econometrista afirma que el análisis de series temporales y los pronósticos están basados en el supuesto de estacionariedad - la constancia a lo largo del tiempo de parámetros como la media, la varianza y la tendencia-, para explicar esto considera el modelo dinámico más simple, el autorregresivo de primer orden

$$
\begin{aligned}
& y_{t}=\alpha+\rho y_{t-1}+e_{t} \\
& E\left(e^{2}\right)=\sigma^{2}
\end{aligned}
$$

donde $e_{\mathrm{t}}$ es una serie temporal de shocks no correlacionados serialmente; bajo el supuesto de estacionariedad los parámetros $\alpha, \rho, \sigma^{2}$ son constantes 
a lo largo del tiempo; si por lo menos uno de ellos ha cambiado en algún momento - la fecha de rompimiento - se dice que ha ocurrido un cambio estructural, en este caso consideran cambios estructurales ocurridos inmediatamente, no paulatinamente.

Si el rompimiento estructural lleva a cambios en el parámetro autorregresivo $\rho$ ello refleja cambios en la correlación serial en $y_{\mathrm{t}}$; por su parte, $\alpha$ controla la media de $y_{\mathrm{t}}$ a través de la relación

$\mathrm{E}\left(\mathrm{y}_{\mathrm{t}}\right)=\mu=\alpha /(1-\rho)$; por último, cambios en $\sigma^{2}$ implican cambios en la volatilidad.

Dentro de los aspectos más destacados en torno a esta literatura de los cambios estructurales para Hansen (2001) están los siguientes tres aspectos: i) la prueba de cambio estructural en un momento desconocido, ii) la estimación del momento del cambio estructural y iii) pruebas para distinguir entre un camino aleatorio y una tendencia temporal segmentada.

En este tercer punto nos explica Hansen (2001): Nelson y Plosser (1982) llegaron a la conclusión de que muchas series macroeconómicas no tienen una tendencia fija a la cual la serie se revierte a lo largo del ciclo económico, la tendencia podría ser movida por shocks aleatorios y la misma permanecería en tal nivel hasta que se presente un nuevo shock aleatorio, por eso se le caracteriza como un camino aleatorio.

Esto derivó en un cúmulo de obras donde examinaban tal situación; no obstante, el trabajo más relevante, en opinión de Hansen (2001), es el de Perron (1989), este autor argumenta que los movimientos de la tendencia podían ser explicados por rompimientos estructurales sencillos parsimoniosos en una tendencia lineal distinta, lo cual es plausible porque el rompimiento en la tendencia produce propiedades de correlación serial similares a las de un camino aleatorio.

Perron (1989) muestra cómo examinar la hipótesis de un camino aleatorio contra el modelo de tendencia rota. Esto lo consigue al estimar una autorregresión lineal, la cual incorpora interacciones de variables dummy para capturar la especificación deseada de la tendencia rota. La hipótesis de la tendencia camino aleatorio implica que la suma de los coeficientes autorregresivos es igual a uno, esto es, una raíz unitaria en el polinomio autorregresivo, de manera que puede ser examinada con un estadístico t-ratio, el cual es no normal, pero Perron provee una teoría de la distribución y los valores críticos.

El artículo de Perron (1989) fue debatido porque se argumentaba es inapropiado especificar la fecha de rompimiento como conocida; así autores como Christiano (1992), Zivot y Andrews (1992), Banerjee, Lumsdaine 
y Stock ((1992), Perron y Vogelsang (1992), sugieren como un procedimiento adecuado seleccionar la fecha de rompimiento con la más grande evidencia contra la hipótesis de camino aleatorio, aquélla con el t-ratio más grande.

Este procedimiento distinto para elegir la fecha de rompimiento requiere distribuciones muestrales diferentes, con valores críticos más grandes, lo cual hace más difícil rechazar la hipótesis nula de un camino aleatorio.

Después desarrollaron las pruebas con dos rompimientos, como la de Lumsdaine y Papell (1997), sus hallazgos iban contra la hipótesis de camino aleatorio. No obstante, explica Hansen (2001), la necesidad de dos rompimientos estructurales reduce la distinción entre modelos de tendencias rotas y caminos aleatorios; a ello agrega que la distinción entre elementos en gran medida se relaciona con la frecuencia de los shocks permanentes sobre la tendencia: en un proceso camino aleatorio tales shocks ocurren frecuentemente, mientras que en un proceso de tendencia rota ocurren con poca frecuencia (uno o dos en la muestra).

\section{Prueba panel de estacionariedad con rompimientos múltiples}

La prueba de estacionariedad con datos panel de Carrion-i-Silvestre et al. (2005) tiene como particularidades las siguientes:

1. Es una versión panel de la prueba univariada de Kwiatkowski, Phillips, Schmidt y Shin (KPSS).

2. La hipótesis nula es de estacionariedad con hasta ocho rompimientos estructurales.

3. La prueba tiene muy alta potencia o probabilidad de rechazar una hipótesis nula falsa.

4. En esta versión se consideran rompimientos que afectan la media y la tendencia.

5. Cada individuo del panel puede tener un número diferente de rompimientos ubicados en fechas distintas.

6. La prueba tiene una distribución límite normal y funciona bien en muestras finitas.

7. Los modelos de la prueba son: sea $\left\{\mathrm{y}_{\mathrm{i}, \mathrm{t}}\right\}$ el conjunto de procesos estocásticos dado por

$\mathrm{y}_{\mathrm{i}, \mathrm{t}}=\alpha_{\mathrm{i}, \mathrm{t}}+\beta_{\mathrm{i}} \mathrm{t}+\varepsilon_{\mathrm{i}, \mathrm{t}}$

$\alpha_{\mathrm{i}, \mathrm{t}}=\Sigma_{\mathrm{k}=1}^{\mathrm{mi}} \theta_{\mathrm{i}, \mathrm{k}} \mathrm{D}\left(\mathrm{T}_{\mathrm{b}, \mathrm{k}}^{\mathrm{i}}\right)_{\mathrm{t}}+\Sigma_{\mathrm{k}=1}^{\mathrm{mi}} \gamma_{\mathrm{i}, \mathrm{k}} \mathrm{DU}_{\mathrm{i}, \mathrm{k}, \mathrm{t}}+\alpha_{\mathrm{i}, \mathrm{t}-1}+v_{\mathrm{i}, \mathrm{t}}$

donde $v_{\mathrm{i}, \mathrm{t}} \sim$ i.i.d. $\left(0, \sigma_{\mathrm{v}, \mathrm{i}}^{2}\right)$ y $\alpha_{\mathrm{i}, 0}=\alpha_{\mathrm{i}}$, una constante, con $i=1, \ldots, \mathrm{N}$ individuos y $t=1, \ldots, \mathrm{T}$ periodos. Las variables ficticias $D\left(\mathrm{~T}_{\mathrm{b}, \mathrm{k}}^{\mathrm{i}}\right)_{\mathrm{t}} \mathrm{y}$ 
$D U_{\mathrm{i}, \mathrm{k}, \mathrm{t}}$ son definidas como $\mathrm{D}\left(\mathrm{T}_{\mathrm{b}, \mathrm{k}}^{\mathrm{i}}\right)_{\mathrm{t}}=1$ para $\mathrm{t}=\mathrm{T}_{\mathrm{b}, \mathrm{k}}^{\mathrm{i}}+1$ y 0 en cualquier otra parte, $\mathrm{y} \mathrm{DU} \mathrm{i}_{\mathrm{i}, \mathrm{k}, \mathrm{t}}=1$ para $\mathrm{t}>\mathrm{T}_{\mathrm{b}, \mathrm{k}}^{\mathrm{i}} \mathrm{y} 0$ en cualquier otra parte, con $\mathrm{T}_{\mathrm{b}, \mathrm{k}}^{\mathrm{i}}$ denotando la $k$-ésima fecha de rompimiento para el $i$-ésimo individuo, $\mathrm{k}=1, \ldots, \mathrm{m}_{\mathrm{i}}, \mathrm{m}_{\mathrm{i}} \geq 1$.

8. El proceso generador de datos (PGD) dado por (1) y (2) descompone $\left\{\mathrm{y}_{\mathrm{i}, \mathrm{t}}\right\}$ como la suma de un camino aleatorio $\left\{\alpha_{\mathrm{i}, \mathrm{t}}\right\}$, y el proceso estocástico, $\left\{\varepsilon_{\mathrm{i}, \mathrm{t}}\right\}$, el se asume satisface las condiciones de regularidad mixtas fuertes definidas en Phillips (1987) y en Phillips y Perron (1988). Además, ellos asumen que $\left\{\varepsilon_{\mathrm{i}, \mathrm{t}}\right\}$ y $\left\{v_{\mathrm{i}, \mathrm{t}}\right\}$ son mutuamente independientes entre las dos dimensiones del conjunto de datos panel. Por lo tanto, la hipótesis nula de estacionariedad panel es equivalente a definir $\sigma_{\mathrm{v}, \mathrm{i}}^{2}=0, \forall i=1, \ldots, \mathrm{N}$, bajo la cual el modelo dado por (1) y (2) se vuelve

$\mathrm{y}_{\mathrm{i}, \mathrm{t}}=\alpha_{\mathrm{i}}+\Sigma_{\mathrm{k}=1}^{\mathrm{mi}} \theta_{\mathrm{i}, \mathrm{k}} \mathrm{DU}_{\mathrm{i}, \mathrm{k}, \mathrm{t}}+\beta_{\mathrm{i}}^{\mathrm{t}}+\sum_{\mathrm{k}=1}^{\mathrm{mi}} \gamma_{\mathrm{i}, \mathrm{k}} \mathrm{DT}_{\mathrm{i}, \mathrm{k}, \mathrm{t}}^{*}+\varepsilon_{\mathrm{i}, \mathrm{t}}$

Con la variable ficticia $\mathrm{DT}_{\mathrm{i}, \mathrm{k}, \mathrm{t}}^{*}=\mathrm{t}-\mathrm{T}_{\mathrm{b}, \mathrm{k}}^{\mathrm{i}}$ para $\mathrm{t}>\mathrm{T}_{\mathrm{b}, \mathrm{k}}^{\mathrm{i}}$ y 0 en cualquier otra parte, $\mathrm{k}=1, \ldots, \mathrm{m}_{\mathrm{i}}, \mathrm{m}_{\mathrm{i}} \geq 1$. El modelo en (3) incluye efectos individuales, efectos rompimiento estructural individual — esto es, desplazamientos en la media causados por rompimientos estructurales, efectos temporales -si $\beta_{\mathrm{i}} \neq 0$ - y efectos rompimiento estructurales temporales - si $\gamma_{\mathrm{i}, \mathrm{k}} \neq 0$, esto es, cuando hay desplazamientos en la tendencia temporal individual. Por lo tanto, cuando $\beta_{\mathrm{i}}=\gamma_{\mathrm{i}, \mathrm{k}}=0$, el modelo en (3) es la contraparte de aquél analizado por Perron y Vogelsang (1992)denotado como modelo 1 por Carrion-i-Silvestre et al. (2005) - mientras que cuando $\beta_{\mathrm{i}} \neq \gamma_{\mathrm{i}, \mathrm{k}} \neq 0$ ellos tienen la especificación dada por el modelo $C$ de Perron (1990), al cual Carrion-i-Silvestre et al (2005) se refieren como el modelo 2.

9. La especificación dada por (3) es lo suficientemente general para permitir las siguientes características: (i) los rompimientos estructurales pueden tener diferentes efectos sobre cada serie de tiempo - los efectos son medidos por $\theta_{\mathrm{i}, \mathrm{k}}$ y $\gamma_{\mathrm{i}, \mathrm{k}}$; (ii) ellos pueden ser localizados en diferentes fechas desde que los autores no restringen las fechas de los rompimientos a satisfacer $\mathrm{T}_{\mathrm{b}, \mathrm{k}}^{\mathrm{i}}=\mathrm{T}_{\mathrm{b}, \mathrm{k}}, \forall \mathrm{i}=1, \ldots, \mathrm{N}$ y (iii) los individuos pueden tener diferente número de rompimientos estructurales $m_{i} \neq m_{j}$, $\forall \mathrm{i} \neq \mathrm{j}, \mathrm{i}, \mathrm{j}=1, \ldots, \mathrm{N}$.

10. La prueba de hipótesis nula de estacionariedad panel sigue la propuesta de Hadri (2000), quien diseñó un estadístico de prueba que es simplemente el promedio de la prueba univariada de estacionariedad en Kwiatkowski et al. (1992). 
11. Para tomar en consideración la dependencia de corte transversal entre individuos o series consideradas, la prueba de Carrion et al. (2005) calcula la distribución bootstrap.

\section{Modelos Autorregresivos y de Rezagos Distribuidos (ARDL)}

Para entender cómo es un modelo ARDL veamos los siguientes elementos considerados por Johnston y Dinardo (2001):

“... un modelo ARDL $(1,1)$ donde la variable dependiente y la explicativa tienen un único retardo

$$
\mathrm{y}_{\mathrm{t}}=\mathrm{m}+\alpha_{1} \mathrm{y}_{\mathrm{t}-1}+\beta_{0} \mathrm{x}_{\mathrm{t}}+\beta_{1} \mathrm{x}_{\mathrm{t}-1}+\varepsilon_{\mathrm{t}} \quad \mathrm{t}=1,2, \ldots, \mathrm{n}
$$

y suponga que $\varepsilon_{\mathrm{t}}$ es ruido blanco. Invirtiendo el polinomio retardado en $y_{\mathrm{t}}$ se obtiene

$$
\mathrm{y}_{\mathrm{t}}=\left(1+\alpha_{1}+\alpha^{2}{ }_{1}+\ldots\right) \mathrm{m}+\left(1+\alpha_{1} \mathrm{~L}+\alpha^{2}{ }_{1} \mathrm{~L}^{2}+\ldots\right)\left(\beta_{0} \mathrm{x}_{\mathrm{t}}+\beta_{1} \mathrm{x}_{\mathrm{t}-1}+\varepsilon_{\mathrm{t}}\right)
$$

"El valor actual de $y$ depende tanto del valor actual como de los pasados de $x$ y de $\varepsilon$.

"Al tomar derivadas parciales se obtiene un conjunto de respuestas dinámicas de $y$ ante cambios en $x$, tanto para el corto, como para el mediano y el largo plazos:

$$
\begin{aligned}
& \frac{\delta y_{t}}{\delta x_{t}}=\beta_{0} \\
& \frac{\delta y_{t+1}}{\delta x_{t}}=\beta_{1}+\alpha_{1} \beta_{0} \\
& \frac{\delta y_{t+2}}{\delta x_{t}}=\alpha_{1} \beta_{1}+\alpha_{1}^{2} \beta_{0}
\end{aligned}
$$

"El efecto a largo plazo de un cambio unitario en $x_{\mathrm{t}}$ se obtiene sumando las derivadas parciales, mientras se satisfaga la condición de estabilidad $\left|\alpha_{1}\right|<1$, y será la suma $\left(\beta_{0}+\beta_{1}\right) /\left(1-\alpha_{1}\right)$.

"Con $x$ en un nivel constante de manera indefinida y con los errores en su nivel esperado cero, $y$ tenderá a un valor constante $y^{-}$definido de la siguiente manera:

$$
\bar{y}=\frac{m}{1-\alpha_{1}}+\frac{\beta_{0}+\beta_{1}}{1-\alpha_{1}} \bar{x}
$$

"Si $y$ y $x$ son los logaritmos naturales de $Y$ y de $X$, respectivamente la relación

$$
\mathrm{Y}=\mathrm{AX} \mathrm{X}^{\gamma}
$$


o en formato logarítmico

$$
\mathrm{y}=\mathrm{a}+\gamma \mathrm{x}
$$

"Para reparametrizar el modelo ARDL $(1,1)$ se debe sustituir $y_{\mathrm{t}}$ por

$y_{\mathrm{t}-1}+\Delta y_{\mathrm{t}}$ y $x_{\mathrm{t}}$ por $x_{\mathrm{t}-1}+\Delta x_{\mathrm{t}}$ para obtener el modelo siguiente:

$\Delta \mathrm{y}_{\mathrm{t}}=\mathrm{m}+\beta_{0} \Delta \mathrm{x}_{\mathrm{t}}-\left(1-\alpha_{1}\right) \mathrm{y}_{\mathrm{t}-1}+\left(\beta_{0}+\beta_{1}\right) \mathrm{x}_{\mathrm{t}-1}+\varepsilon_{\mathrm{t}}$

"El cual al ser reformulado mediante las ecuaciones de elasticidades lleva a

$$
\Delta \mathrm{y}_{\mathrm{t}}=\beta_{0} \Delta \mathrm{x}_{\mathrm{t}}-\left(1-\alpha_{1}\right)\left[\mathrm{y}_{\mathrm{t}-1}-\mathrm{a}-\gamma \mathrm{x}_{\mathrm{t}-1}\right]+\varepsilon_{\mathrm{t}}
$$

"La expresión anterior es un ejemplo de un modelo de corrección de error (MCE), en la cual el cambio en $y$ es la suma de dos componentes: el primero proporcional al cambio sucedido en $x$ y el segundo es una corrección parcial a la desviación de $y_{\mathrm{t}-1}$ con respecto al valor de equilibrio correspondiente a $x_{\mathrm{t}-1}$. El factor entre corchetes es llamado error de equilibrio, el cual si es positivo implica una corrección hacia abajo y viceversa (Johnston y Dinardo, 2001: 282 y 283$)$.

Giles (2013) explica que la utilidad de este modelo es permitir evaluar si existen relaciones de largo plazo entre las series de tiempo estudiadas cuando tenemos dudas del orden de integración de las mismas, pero el mismo no es igual o superior a dos. Con el modelo estimado estable se hacen las pruebas de límites (Bounds tests) propuestas por Pesaran et al. (2001), estas pruebas evalúan si los coeficientes de las variables son estadísticamente iguales a cero en el modelo estimado, para ello estima un valor critico que sigue una distribución $F$. Si el estadístico crítico es mayor al límite superior se concluye que existe una relación de largo plazo, si está por debajo del límite inferior significa que no la hay y si su valor se encuentra al interior del intervalo entonces la prueba no es concluyente.

\section{Datos de Migración NETA INTERNACIONAL, INMIGRACiÓN Y EMIGRACIÓN DE MÉXICO POR SEXo EN EL PERIOdo 1960-2010}

García-Guerreo (2016) aplica para datos de México un método para determinar el índice de migración neta modificado, la inmigración y emigración internacionales a partir de los modelos de Lee (1993) y de Lee y Carter (1992), el cual deriva niveles de migración sostenibles a partir de considerar toda la dinámica demográfica, para no llegar a valores pronosticados negativos de la población total. El método lo aplica a datos de la Sociedad 
Mexicana de Demografía (SOMEDE, 2011) de flujos netos migratorios, de inmigración y emigración, por sexo y edad (los cuales no están disponibles).

García-Guerrero señala:

Tal patrón de edad indica que la migración tiene un carácter netamente laboral y debido a la reunificación familiar se ha intensificado en los años iniciales de la vida. Además, el parámetro de intensidad refleja que la migración neta es fuerte en las edades laborales. Por último, el parámetro tendencial apunta que tal tendencia es descendente, pero es muy difícil afirmar que tal tendencia continuará cayendo continuamente. Esto debido a la alta volatilidad del fenómeno migratorio y al reciente cambio de tendencia (García-Guerrero, 2016: 122).

García-Guerrero (2016) concluye que el mejor modelo de series de tiempo estimado y pronosticado para el índice modificado de la migración neta para hốmbres es un camino aleatorio, $\operatorname{ARIMA}(0,1,0)$, esto es $\mathrm{z}_{\mathrm{t}}=\mathrm{z}_{\mathrm{t}-1}+\mathrm{e}_{\mathrm{t}}$, con $\quad=0.0617$; por otra parte, para mujeres el mejor modelo es un proceso promedio móvil integrado de orden uno, esto es, ARIMA $(0,1,1), \mathrm{z}_{\mathrm{t}}=\mathrm{z}_{\mathrm{t}-1}+\mathrm{e}_{\mathrm{t}}+\theta \mathrm{e}_{\mathrm{t}-1}=\mathrm{z}_{\mathrm{t}-1}+\mathrm{e}_{\mathrm{t}}-0.2783 \mathrm{e}_{\mathrm{t}-1}$, con $\hat{\sigma}_{\mathrm{et}}^{2}=0.06784$, el cual implica que en el horizonte de proyección la migración neta permanece en la mediana sin cambio.

Para la emigración y la inmigración el límite inferior para ambos sexos es cero; por su parte, el límite superior en hombres es un millón y en mujeres es 800 mil. El mejor modelo para el índice de emigración modificado en ambos sexos es un camino aleatorio, lo mismo para el índice de inmigración modificado.

Como los datos sobre migración de la SOMEDE (2011) no están disponibles hicimos uso del software libre Engauge Digitizer 9.8 de Mitchell Mark, el cual permite la extracción eficiente de los datos numéricos exactos a partir de imágenes de gráficos, al convertir pixeles en números, un proceso denominado "graficación inversa". En este caso, los valores más cercanos a cada año están presentes en la Tabla 1. 


\section{Estacionariedad, rompimientos y tendencias de la migración internacional de México/Z.S. HERNÁNDEZ y T. SERRANO}

Tabla 1: Estimaciones de índices de migración neta, inmigración y emigración modificados de GarcíaGuerrero (2016) obtenidos mediante Engauge Digitizer 9.8

\begin{tabular}{|c|c|c|c|c|c|c|}
\hline Año & $\begin{array}{r}\text { Índice migración } \\
\text { neta femenina } \\
\text { modificado }\end{array}$ & $\begin{array}{r}\text { Índice migración } \\
\text { neta masculina } \\
\text { modificado }\end{array}$ & $\begin{array}{r}\text { Emigración } \\
\text { femenina }\end{array}$ & $\begin{array}{r}\text { Emigración } \\
\text { masculina }\end{array}$ & $\begin{array}{l}\text { Inmigración } \\
\text { femenina* }\end{array}$ & $\begin{array}{l}\text { Inmigración } \\
\text { masculina* }\end{array}$ \\
\hline 1960 & 90.57 & 110.45 & 19.49 & 17.12 & 71.08 & 93.33 \\
\hline 1961 & 90.51 & 111.60 & 21.23 & 18.99 & 69.28 & 92.61 \\
\hline 1962 & 90.46 & 111.71 & 20.06 & 16.85 & 70.40 & 94.86 \\
\hline 1963 & 90.40 & 111.80 & 21.22 & 16.71 & 69.18 & 95.09 \\
\hline 1964 & 90.35 & 111.90 & 21.80 & 17.34 & 68.55 & 94.56 \\
\hline 1965 & 93.57 & 1160 & 17.72 & 16.44 & 75.85 & 99.56 \\
\hline 1966 & 91.87 & 112.10 & 24.71 & 17.94 & 67.16 & 94.16 \\
\hline 1967 & 88.53 & 109.20 & 26.45 & 20.25 & 62.08 & 88.95 \\
\hline 1968 & 85.18 & 106.30 & 29.94 & 25.00 & 55.24 & 81.30 \\
\hline 1969 & 80.20 & 100.40 & 32.84 & 30.57 & 47.36 & 69.83 \\
\hline 1970 & 67.00 & 83.50 & 48.00 & 50.00 & 19.00 & 33.50 \\
\hline 1971 & 67.02 & 78.60 & 54.99 & 59.64 & 12.03 & 18.96 \\
\hline 1972 & 57.03 & 70.71 & 59.06 & 65.22 & -2.03 & 5.49 \\
\hline 1973 & 52.04 & 64.80 & 63.72 & 71.61 & -11.68 & -6.81 \\
\hline 1974 & 50.34 & 58.90 & 66.05 & 76.36 & -15.71 & -17.46 \\
\hline 1975 & 65.04 & 90.00 & 63.13 & 77.03 & 1.91 & 12.97 \\
\hline 1976 & 61.73 & 84.10 & 71.28 & 84.24 & -9.55 & -0.14 \\
\hline 1977 & 58.38 & 79.20 & 74.78 & 89.81 & -16.40 & -10.61 \\
\hline 1978 & 55.04 & 75.30 & 75.94 & 93.75 & -20.90 & -18.45 \\
\hline 1979 & 56.63 & 71.40 & 78.26 & 96.06 & -21.63 & -24.66 \\
\hline 1980 & 51.64 & 59.50 & 75.93 & 100.00 & -24.29 & -40.50 \\
\hline 1981 & 46.66 & 53.60 & 78.84 & 106.39 & -32.18 & -52.79 \\
\hline 1982 & 46.60 & 49.70 & 82.33 & 111.96 & -35.73 & -62.26 \\
\hline 1983 & 44.90 & 44.80 & 85.24 & 116.71 & -40.34 & -71.91 \\
\hline 1984 & 39.92 & 36.90 & 91.06 & 122.29 & -51.14 & -85.39 \\
\hline 1985 & 23.43 & 9.00 & 100.96 & 137.63 & -77.53 & -128.63 \\
\hline 1986 & 11.87 & -3.90 & 113.2 & 153.81 & -101.33 & -157.71 \\
\hline 1987 & -2.97 & -17.80 & 126.59 & 169.15 & -129.56 & -186.95 \\
\hline 1988 & -14.53 & -29.70 & 140.57 & 187.7 & -155.10 & -217.4 \\
\hline 1989 & -35.94 & -39.60 & 151.64 & 194.16 & -187.58 & -233.76 \\
\hline 1990 & -75.43 & -61.49 & 200.00 & 225.00 & -275.43 & -286.49 \\
\hline 1991 & -86.99 & -73.40 & 219.81 & 238.73 & -306.8 & -312.13 \\
\hline 1992 & -96.90 & -85.30 & 227.96 & 251.63 & -324.86 & -336.93 \\
\hline 1993 & -105.17 & -96.20 & 239.03 & 263.72 & -344.20 & -359.92 \\
\hline 1994 & -110.16 & -111.10 & 245.43 & 281.52 & -355.59 & -392.62 \\
\hline 1995 & -100.36 & -136.00 & 247.18 & 316.44 & -347.54 & -452.44 \\
\hline 1996 & -110.27 & -156.90 & 254.17 & 334.24 & -364.44 & -491.14 \\
\hline 1997 & -118.55 & -178.80 & 263.48 & 356.93 & -382.03 & -535.73 \\
\hline 1998 & -125.17 & -189.40 & 268.72 & 375.54 & -393.89 & -564.94 \\
\hline 1999 & -136.73 & -199.60 & 270.47 & 403.94 & -407.20 & -603.54 \\
\hline 2000 & -240.29 & -300.50 & 371.27 & 478.84 & -611.56 & -779.34 \\
\hline 2001 & -143.41 & -184.40 & 275.12 & 355.57 & -418.53 & -539.97 \\
\hline 2002 & -112.26 & -158.30 & 245.98 & 338.31 & -358.24 & -496.61 \\
\hline 2003 & -132.03 & -178.20 & 259.38 & 358.55 & -391.41 & -536.75 \\
\hline 2004 & -125.52 & -209.40 & 255.30 & 385.32 & -380.82 & -594.72 \\
\hline 2005 & -50.00 & -91.00 & 243.64 & 386.00 & -293.64 & -477.00 \\
\hline 2006 & -2.41 & 0.10 & 200.52 & 304.34 & -202.93 & -304.24 \\
\hline 2007 & 25.46 & 39.20 & 167.89 & 270.78 & -142.43 & -231.58 \\
\hline 2008 & 45.12 & 68.30 & 149.82 & 240.48 & -104.70 & -172.18 \\
\hline 2009 & 68.06 & 120.40 & 128.20 & 184.07 & -60.14 & -63.67 \\
\hline 2010 & 66.36 & 122.52 & 130.00 & 185.54 & -63.64 & -63.02 \\
\hline
\end{tabular}

* Obtenido como la diferencia entre la migración neta y la emigración por sexo respectiva.

Fuente: elaboración propia a partir de García-Guerrero (2016) y Engauge Digitizer 9.8. 


\section{AnÁlisis ECONOMÉTRICo}

\section{Prueba panel de estacionariedad con rompimientos múltiples}

Al aplicar la prueba de estacionariedad panel con rompimientos múltiples se encuentra en las seis series migratorias dos rompimientos bajo el criterio LWZ o criterio de selección de Schwarz modificado; para las series femeninas el primer rompimiento ocurre a finales de años ochenta y el segundo rompimiento en el año 1999; para las series masculinas el primer rompimiento es detectado en el primer lustro de la década de los ochenta (entre cinco y seis años antes que el primer rompimiento femenino) y el segundo rompimiento es el año 1999, mismo año del segundo rompimiento femenino (Tabla 2).

Tabla 2: Estimación del número de rompimientos estructurales y año del mismo

\begin{tabular}{lcr}
\hline Variable & \multicolumn{2}{c}{ Año de rompimiento } \\
\cline { 2 - 3 } & Primero & Segundo \\
\hline Migración neta femenina & 1988 & 1999 \\
Migración neta masculina & 1982 & 1999 \\
Emigración femenina & 1989 & 1999 \\
Emigración masculina & 1984 & 1999 \\
Inmigración femenina & 1989 & 1999 \\
Inmigración masculina & 1983 & 1999 \\
\hline
\end{tabular}

Fuente: Código en Gauss de Carrion-i-Silvestre et al. (2005) para ocho rompimientos.

Otro aspecto a considerar es un intervalo de confianza para cada una de las fechas de rompimiento, en este caso si consideramos un nivel de confianza de 95 por ciento tenemos que los límites inferiores se extienden uno o dos años abajo del rompimiento correspondiente, mientras los límites superiores también están un año o dos como máximo de la fecha de rompimiento (Tabla 3).

Las regresiones donde se determina mediante variables dummy si el rompimiento es en el intercepto, en la pendiente o en ambos son mostrados en las siguientes seis tablas y seis figuras, de ellas cabe establecer que el primer rompimiento para hombres se debe esencialmente a dos factores: el boom demográfico de México de nacimientos en los sesenta y la crisis económica de los años ochenta; estos dos factores principalmente llevaron a una mayor salida neta de personas de México, primero para los hombres (años 1982, 1983 y 1984) y con cierto rezago temporal para las mujeres (1988 y 1999), como se puede concluir de la Figura 3. 
Tabla 3: Intervalo de confianza de cada rompimiento por tipo de flujo (año)

\begin{tabular}{lrrr}
\hline \multicolumn{1}{c}{ Rompimiento por tipo de flujo } & $\begin{array}{c}\text { Lím. } \\
\text { inferior }\end{array}$ & Rompim. & $\begin{array}{r}\text { Lím. } \\
\text { superior }\end{array}$ \\
\hline Primer rompimiento flujo neto femenino & 1986 & 1988 & 1990 \\
Segundo rompimiento flujo neto femenino & 1997 & 1999 & 2000 \\
Primer rompimiento flujo neto masculino & 1980 & 1982 & 1983 \\
Segundo rompimiento flujo neto masculino & 1998 & 1999 & 2000 \\
Primer rompimiento emigración femenina & 1987 & 1989 & 1990 \\
Segundo rompimiento emigración femenina & 1997 & 1999 & 2000 \\
Primer rompimiento emigración masculina & 1982 & 1984 & 1985 \\
Segundo rompimiento emigración masculina & 1998 & 1999 & 2000 \\
Primer rompimiento inmigración femenina & 1987 & 1989 & 1990 \\
Segundo rompimiento inmigración femenina & 1998 & 1999 & 2000 \\
Primer rompimiento inmigración masculina & 1981 & 1983 & 1984 \\
Segundo rompimiento inmigración masculina & 1998 & 1999 & 2000 \\
\hline
\end{tabular}

Fuente: Código en Gauss de Carrion-i-Silvestre et al. (2005) para ocho rompimientos.

Con respecto al segundo rompimiento, el cual implica un cambio en el signo de la pendiente de los flujos de emigración positivo a negativo sólo podemos afirmar que es previo a los acontecimientos de terrorismo de 2001 en Estados Unidos.

Al ser los estadísticos calculados (Tabla 4) menores que los valores críticos bootstrap con varianzas homogénea y heterogénea (Tabla 5) no podemos rechazar la hipótesis nula de estacionariedad con rompimientos. Esto nos lleva a concluir que no tenemos caminos aleatorios en las series. La confusión se debe a la presencia de rompimientos en las series como explica Hansen (2001). ${ }^{1}$

\footnotetext{
${ }^{1}$ En un camino aleatorio sin deriva el mejor pronóstico para $l$ periodos es la última observación disponible $y_{\mathrm{T}}$, y la varianza del error de pronóstico para $l$ periodos es $l \sigma^{2}$ Si se trabaja con un camino aleatorio con deriva o rumbo denotado por $d$, donde existe una tendencia ascendente o descendente de la serie $y_{t}$, entonces el mejor pronóstico está dado por $\mathrm{y}_{\mathrm{T}}+l d$; y la varianza del error de pronóstico es nuevamente $l \sigma_{\varepsilon}^{2}$. Lo anterior según Pyndick y Rubinfeld (2001).
} 





Tabla 4: Estadísticos calculados de Hadri con varianzas homogénea y heterogénea para seis series de flujos migratorios de México, 1960-2010

Estadístico calculado de Hadri con varianza homogénea 4.2736451

Estadístico calculado de Hadri con varianza heterogénea 4.8751563

Fuente: Código en Gauss de Carrion-i-Silvestre et al. (2005) para ocho rompimientos.

Tabla 5: Estadísticos críticos bootstrap de estacionariedad con rompimientos y varianzas homogénea y heterogénea

\begin{tabular}{lr}
\hline Homogéneo & \\
\hline Nivel de confianza & Estadístico crítico bootstrap \\
\hline 0.90 & 9.12 \\
0.95 & 11.63 \\
0.98 & 13.92 \\
0.99 & 16.35 \\
\hline
\end{tabular}

\begin{tabular}{rr}
\multicolumn{1}{l}{ Heterogéneo } & \\
\hline Nivel de confianza & Estadístico crítico bootstrap \\
\hline 0.90 & 10.93 \\
0.95 & 13.52 \\
0.98 & 15.88 \\
0.99 & 18.94 \\
\hline
\end{tabular}

Fuente: Código en Gauss de Carrion-i-Silvestre et al. (2005) para ocho rompimientos.

\section{Modelo ARDL}

Para examinar si existe una relación de largo plazo entre las variables Migración Neta Femenina (MNF) y Migración Neta Masculina (MNM) para México con datos del periodo 1960-2010, recurrimos a la técnica econométrica ARDL, la cual plantea en su hipótesis nula la no existencia de tal relación, y en hipótesis alternativa la existencia de la misma entre las variables. Cabe aclarar que no fue necesario incorporar variables ficticias o dummy en la estimación para considerar los rompimientos de la migración (Tabla 6).

El ajuste logrado entre la migración neta femenina observada (actual) y la migración neta femenina estimada (fitted) es muy alto (Figura 4); asimismo, encontramos que los residuos tienen una volatilidad muy similar a lo largo de todo el periodo.

Para confirmar que el modelo anterior, $\operatorname{ARDL}(8,8)$ está bien especificado fueron aplicadas varias pruebas de no correlación serial (función de autocorrelación y de autocorrelación parcial de los residuos y de los residuos al cuadrado, el estadístico $Q$ de Box-Pierce,la prueba de Breusch-Godfrey) y no se encontró tal problema. 
Tabla 6: Modelo ARDL $(8,8)$

Variable dependiente: MNF

Método: ARDL

Muestra (ajustada): 19682010

Observaciones: 43 después del ajuste

Máximo de rezagos dependientes: 8 (selección automática)

Método de selección de (AIC)

Regresores dinámicos ( 8 rezagos, automático): MNM

Regresores fijos:

Número de modelos evaluados: 72

Modelo seleccionado: ARDL $(8,8)$

\begin{tabular}{|c|c|c|c|c|}
\hline Variable & Coeficiente & Error estándar & $t$-calculado & Prob.* \\
\hline MNF(-1) & 1.006262 & 0.188149 & 5.348212 & 0.0000 \\
\hline $\operatorname{MNF}(-2)$ & -0.015466 & 0.284666 & -0.054331 & 0.9571 \\
\hline $\operatorname{MNF}(-3)$ & -0.213009 & 0.266916 & -0.798037 & 0.4321 \\
\hline $\operatorname{MNF}(-4)$ & -0.489704 & 0.265040 & -1.847662 & 0.0761 \\
\hline $\operatorname{MNF}(-5)$ & -0.161338 & 0.248283 & -0.649816 & 0.5215 \\
\hline MNF(-6) & 0.475321 & 0.218136 & 2.179013 & 0.0386 \\
\hline $\operatorname{MNF}(-7)$ & 0.124332 & 0.339059 & 0.366698 & 0.7168 \\
\hline $\operatorname{MNF}(-8)$ & -0.571067 & 0.256178 & -2.229181 & 0.0347 \\
\hline MNM & 0.865278 & 0.044078 & 19.63081 & 0.0000 \\
\hline MNM(-1) & -0.797022 & 0.180299 & -4.420547 & 0.0002 \\
\hline MNM(-2) & -0.006307 & 0.246253 & -0.025612 & 0.9798 \\
\hline MNM(-3) & 0.202314 & 0.240485 & 0.841277 & 0.4079 \\
\hline MNM(-4) & 0.122844 & 0.227607 & 0.539723 & 0.5940 \\
\hline MNM(-5) & 0.268036 & 0.171711 & 1.560976 & 0.1306 \\
\hline MNM(-6) & -0.315858 & 0.158008 & -1.999004 & 0.0562 \\
\hline MNM(-7) & -0.189987 & 0.294384 & -0.645371 & 0.5243 \\
\hline MNM(-8) & 0.535155 & 0.223821 & 2.390997 & 0.0243 \\
\hline
\end{tabular}

* Nota: Valores de probabilidad y cualquier prueba subsecuente no se considera para el modelo.

Fuente: elaboración propia.

Otra característica deseada en los residuos para hacer inferencia es la normalidad, al aplicar la prueba de Jarque-Bera no rechazamos la hipótesis nula de normalidad (Figura 5).

Al aplicar la prueba Bounds Test de Pesaran et al. (2001) podemos rechazar la hipótesis nula de no existencia de relación de largo plazo entre migración neta femenina y migración neta masculina en todos los niveles de significancia, al ser el estadístico calculado (7.816535) superior a todos los valores críticos sin importar el nivel de significancia (Tabla 7).

Para establecer la relación de largo plazo examinamos la ecuación de cointegración; en específico, el coeficiente respectivo, el cual es altamente negativo y significativo (Tabla 8). 
Estacionariedad, rompimientos y tendencias de la migración internacional de México/Z.S. HERNÁNDEZ y T. SERRANO

Figura 4: Ajuste entre MNF observada y MNF estimada según modelo ARDL $(8,8)$

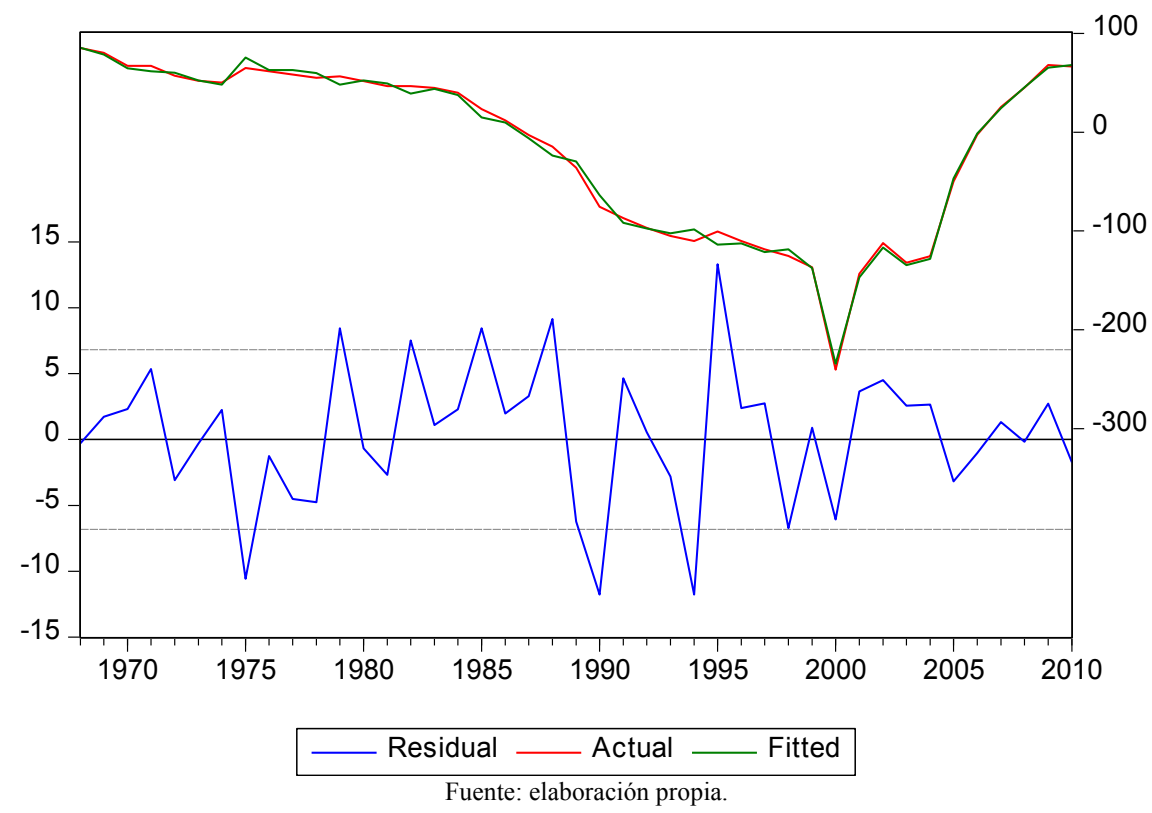

Figura 5: Histograma y prueba de normalidad de Jarque-Bera a los residuos del modelo ARDL $(8,8)$

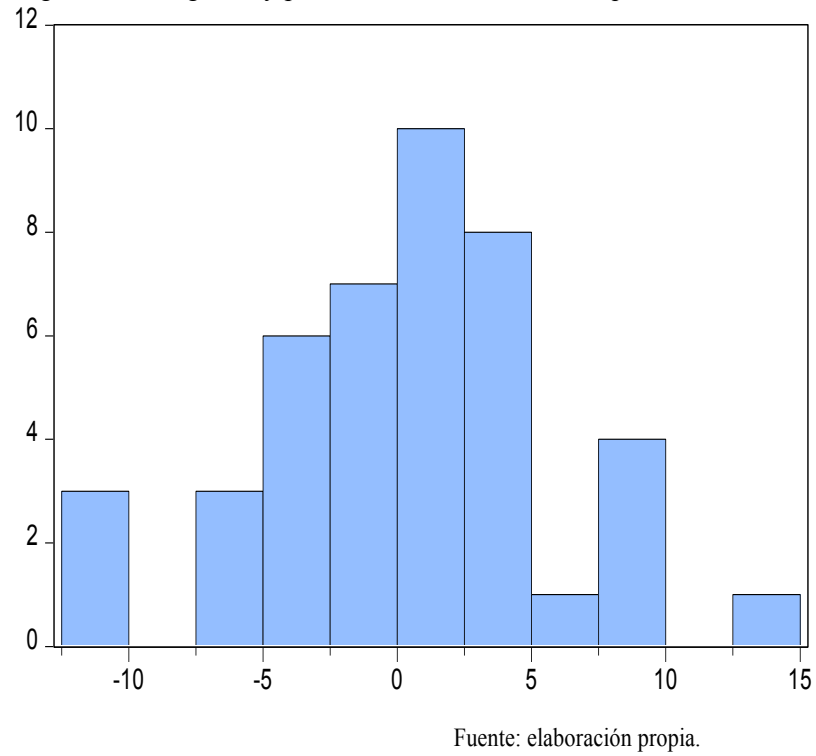

Series: Residuals

Sample 19682010

Observations 43

Mean $\quad 0.371578$

Median $\quad 1.083205$

Maximum $\quad 13.29991$

Minimum $\quad-11.78105$

Std. Dev. $\quad 5.351082$

Skewness $\quad-0.247081$

Kurtosis $\quad 3.341949$

Jarque-Bera $\quad 0.647016$

Probability $\quad 0.723606$

Fuente: elaboración propia. 
Tabla 7: Prueba ARDL de límites

Muestra: 19682010

Observaciones incluidas: 43

Hipótesis nula: No existe relación de largo plazo

\begin{tabular}{lrr}
\hline Estadístico de prueba & Valor & $\mathrm{K}$ \\
\hline Estadístico $F$ & 7.816535 & 1 \\
\hline Significancia & & Límites de los valores críticos \\
\hline $10 \%$ & $\mathrm{I}_{0}$ Bound & $\mathrm{I}_{1}$ Bound \\
$5 \%$ & 2.44 & 3.28 \\
$2.5 \%$ & 3.15 & 4.11 \\
$1 \%$ & 3.88 & 4.92 \\
\hline
\end{tabular}

Fuente: elaboración propia.

Tabla 8: Modelo ARDL en diferencias D(MNF)

\begin{tabular}{lrrrl}
\hline Variable & Coeficiente & Error estándar & $t$-calculado & Prob. \\
\hline D(MNF(-1)) & 0.850932 & 0.224018 & 3.798503 & 0.0008 \\
D(MNF(-2)) & 0.835466 & 0.255377 & 3.271503 & 0.0030 \\
D(MNF(-3)) & 0.622457 & 0.231550 & 2.688217 & 0.0124 \\
D(MNF(-4)) & 0.132752 & 0.206941 & 0.641498 & 0.5268 \\
D(MNF(-5)) & -0.028586 & 0.166121 & -0.172079 & 0.8647 \\
D(MNF(-6)) & 0.446735 & 0.190872 & 2.340493 & 0.0272 \\
D(MNF(-7)) & 0.571067 & 0.256178 & 2.229181 & 0.0347 \\
D(MNM) & 0.865278 & 0.044078 & 19.63081 & 0.0000 \\
D(MNM(-1)) & -0.616197 & 0.176112 & -3.498898 & 0.0017 \\
D(MNM(-2)) & -0.622504 & 0.194491 & -3.200692 & 0.0036 \\
D(MNM(-3)) & -0.420190 & 0.191812 & -2.190632 & 0.0376 \\
D(MNM(-4)) & -0.297346 & 0.159029 & -1.869761 & 0.0728 \\
D(MNM(-5)) & -0.029310 & 0.139368 & -0.210304 & 0.8351 \\
D(MNM(-6)) & -0.345168 & 0.181346 & -1.903369 & 0.0681 \\
D(MNM(-7)) & -0.535155 & 0.223821 & -2.390997 & 0.0243 \\
MNM(-1) & 0.684453 & 0.174736 & 3.917081 & 0.0006 \\
MNF(-1) & -0.844669 & 0.213909 & -3.948737 & 0.0005 \\
\hline
\end{tabular}

Fuente: elaboración propia.

La conclusión cuantitativa del largo plazo es: por cada un mil personas del sexo masculino registradas en la migración neta se tendrán 810 mujeres en la variable migración neta (Tabla 9). 
Tabla 9: Modelo ARDL forma cointegración y largo plazo

\begin{tabular}{|c|c|c|c|c|}
\hline $\begin{array}{l}\text { Variable deper } \\
\text { Modelo selecc } \\
\text { Muestra: } 1960 \\
\text { Observaciones }\end{array}$ & $\begin{array}{l}\text { te original: } 1 \\
\text { do: ARDL( } 8 \text {, } \\
0 \\
\text { uidas: } 43\end{array}$ & $\begin{array}{l}\text { INF } \\
8)\end{array}$ & & \\
\hline \multicolumn{5}{|c|}{$\begin{array}{l}\text { Forma cointegración } \\
\end{array}$} \\
\hline Variable & Coeficiente & Error estándar & $t$-calculado & Prob. \\
\hline$\overline{\mathrm{D}(\mathrm{MNF}(-1))}$ & 0.850932 & 0.216413 & 3.931986 & 0.0006 \\
\hline $\mathrm{D}(\mathrm{MNF}(-2))$ & 0.835466 & 0.250056 & 3.341115 & 0.0025 \\
\hline $\mathrm{D}(\mathrm{MNF}(-3))$ & 0.622457 & 0.226244 & 2.751258 & 0.0107 \\
\hline $\mathrm{D}(\mathrm{MNF}(-4))$ & 0.132752 & 0.201190 & 0.659834 & 0.5152 \\
\hline $\mathrm{D}(\mathrm{MNF}(-5))$ & -0.028586 & 0.162264 & -0.176169 & 0.8615 \\
\hline $\mathrm{D}(\mathrm{MNF}(-6))$ & 0.446735 & 0.180506 & 2.474909 & 0.0202 \\
\hline $\mathrm{D}(\mathrm{MNF}(-7))$ & 0.571067 & 0.250200 & 2.282444 & 0.0309 \\
\hline $\mathrm{D}(\mathrm{MNM})$ & 0.865278 & 0.041488 & 20.856310 & 0.0000 \\
\hline $\mathrm{D}(\mathrm{MNM}(-1))$ & -0.616197 & 0.171275 & -3.597701 & 0.0013 \\
\hline $\mathrm{D}(\mathrm{MNM}(-2))$ & -0.622504 & 0.190707 & -3.264187 & 0.0031 \\
\hline $\mathrm{D}(\mathrm{MNM}(-3))$ & -0.420190 & 0.187831 & -2.237065 & 0.0341 \\
\hline $\mathrm{D}(\mathrm{MNM}(-4))$ & -0.297346 & 0.150555 & -1.974993 & 0.0590 \\
\hline $\mathrm{D}(\mathrm{MNM}(-5))$ & -0.029310 & 0.130987 & -0.223762 & 0.8247 \\
\hline D(MNM(-6)) & -0.345168 & 0.159336 & -2.166282 & 0.0396 \\
\hline D(MNM(-7)) & -0.535155 & 0.219469 & -2.438411 & 0.0219 \\
\hline CointEq(-1) & -0.844669 & 0.209638 & -4.029186 & 0.0004 \\
\hline \multicolumn{5}{|c|}{ Ecuación de cointegración $=$ MNF - $(0.8103 * M N M)$} \\
\hline \multicolumn{5}{|c|}{ Coeficientes de largo plazo } \\
\hline Variable & Coeficiente & Error estándar & $t$-calculado & Prob. \\
\hline MNM & 0.810321 & 0.017703 & 45.773028 & 0.0000 \\
\hline
\end{tabular}

Fuente: elaboración propia.

\section{Pronóstico Lineal}

A partir de los modelos basados en variables dummy, los cuales determinan los rompimientos en las seis series migratorias, construimos proyecciones para el periodo 2011-2040. Estas son válidas siempre y cuando no se presente un nuevo rompimiento o rompimientos en la pendiente ni en la tendencia temporal. Si no se presenta ningún rompimiento estos son los pronósticos adecuados en el largo plazo para la migración neta, la emigración y la inmigración por sexo (Figura 6 y Tabla 10). 


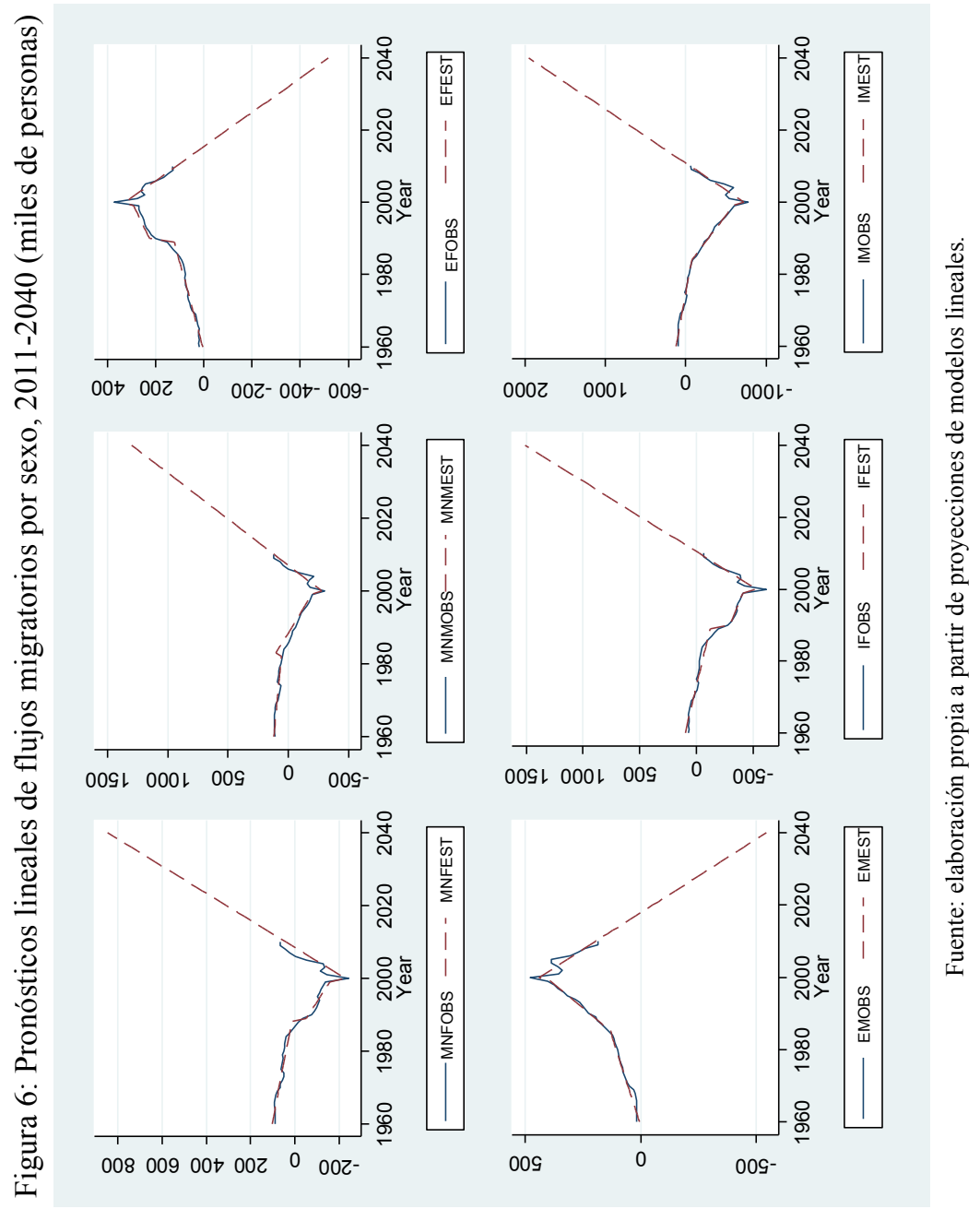


Tabla 10: Flujos migratorios estimados y pronosticados por sexo, 2011 (miles de personas)

\begin{tabular}{|c|c|c|c|c|c|c|}
\hline Año & MNFEST & MNMEST & EFEST & EMEST & IFEST & IMEST \\
\hline 2011 & 68.97 & 154.79 & 94.09 & 169.66 & 30.70 & 14.32 \\
\hline 2012 & 95.75 & 194.21 & 72.99 & 145.09 & 81.70 & 81.23 \\
\hline 2013 & 122.54 & 233.63 & 51.90 & 120.53 & 132.70 & 148.15 \\
\hline 2014 & 149.32 & 273.06 & 30.81 & 95.96 & 183.69 & 215.07 \\
\hline 2015 & 176.10 & 312.48 & 9.71 & 71.39 & 234.69 & 281.98 \\
\hline 2016 & 202.88 & 351.90 & -11.38 & 46.82 & 285.69 & 348.90 \\
\hline 2017 & 229.67 & 391.33 & -32.47 & 22.25 & 336.68 & 415.82 \\
\hline 2018 & 256.45 & 430.75 & -53.57 & -2.31 & 387.68 & 482.74 \\
\hline 2019 & 283.23 & 470.17 & -74.66 & -26.88 & 438.68 & 549.65 \\
\hline 2020 & 310.02 & 509.60 & -95.75 & -51.45 & 489.67 & 616.57 \\
\hline 2021 & 336.80 & 549.02 & -116.85 & -76.02 & 540.67 & 683.49 \\
\hline 2022 & 363.58 & 588.44 & -137.94 & -100.59 & 591.67 & 750.41 \\
\hline 2023 & 390.36 & 627.86 & -159.03 & -125.16 & 642.66 & 817.32 \\
\hline 2024 & 417.15 & 667.29 & -180.13 & -149.72 & 693.66 & 884.24 \\
\hline 2025 & 443.93 & 706.71 & -201.22 & -174.29 & 744.66 & 951.16 \\
\hline 2026 & 470.71 & 746.13 & -222.31 & -198.86 & 795.65 & 1018.08 \\
\hline 2027 & 497.50 & 785.56 & -243.41 & -223.43 & 846.65 & 1084.99 \\
\hline 2028 & 524.28 & 824.98 & -264.50 & -248.00 & 897.65 & 1151.91 \\
\hline 2029 & 551.06 & 864.40 & -285.59 & -272.57 & 948.64 & 1218.83 \\
\hline 2030 & 577.84 & 903.82 & -306.69 & -297.13 & 999.64 & 1285.75 \\
\hline 2031 & 604.63 & 943.25 & -327.78 & -321.70 & 1050.64 & 1352.66 \\
\hline 2032 & 631.41 & 982.67 & -348.87 & -346.27 & 1101.63 & 1419.58 \\
\hline 2033 & 658.19 & 1022.09 & -369.97 & -370.84 & 1152.63 & 1486.50 \\
\hline 2034 & 684.98 & 1061.52 & -391.06 & -395.41 & 1203.63 & 1553.41 \\
\hline 2035 & 711.76 & 1100.94 & -412.15 & -419.98 & 1254.62 & 1620.33 \\
\hline 2036 & 738.54 & 1140.36 & -433.25 & -444.54 & 1305.62 & 1687.25 \\
\hline 2037 & 765.33 & 1179.78 & -454.34 & -469.11 & 1356.62 & 1754.17 \\
\hline 2038 & 792.11 & 1219.21 & -475.43 & -493.68 & 1407.61 & 1821.08 \\
\hline 2039 & 818.89 & 1258.63 & -496.53 & -518.25 & 1458.61 & 1888.00 \\
\hline 2040 & 845.67 & 1298.05 & -517.62 & -542.82 & 1509.61 & 1954.92 \\
\hline
\end{tabular}

Fuente: elaboración propia a partir de proyecciones de modelos lineales.

¿Cuándo se presentará el siguiente rompimiento? Ello depende de muy diversos factores, algunos asociados con la situación económica de México y Estados Unidos, otros con cuestiones demográficas y de política migratoria; no obstante, es casi imposible predecir la fecha de tal rompimiento.

\section{Dinámica}

Como concluimos del modelo $\operatorname{ARDL}(8,8)$ : existe una relación de largo plazo entre la migración neta femenina (MNF) y la migración neta mascu- 
lina (MNM) en el periodo 1960-2010, donde esta última variable se mueve primero que aquélla, nuestra variable dependiente es MNF y la independiente es la MNM, lo cual se basa en mucho en la reunificación familiar y en las cuestiones de migración laboral.

Así, una vez confirmado que todas las series temporales migratorias son estacionarias con rompimientos pretendemos definir un modelo ajustado a los datos observados de la MNM, con el cual se pueda pronosticar tal variable a un horizonte hasta el año 2040, luego a partir de tal insumo incorporado en el modelo $\operatorname{ARDL}(8,8)$ obtener el pronóstico de la MNF para el periodo 2011-2040. Este pronóstico asume que los rompimientos han definido una especie de ciclo de largo plazo.

Con esta idea en mente, buscamos identificar y calcular modelos de series de tiempo para las seis variables migratorias: migración neta femenina, migración neta masculina, emigración femenina, emigración masculina, inmigración femenina e inmigración masculina para el periodo 1960-2010; los modelos estimados son: recorrido aleatorio, tendencia lineal, media móvil, suavizado exponencial simple, $\operatorname{ARMA}(0,0), \operatorname{ARMA}(1,0), \operatorname{ARMA}(2,1)$, $\operatorname{ARMA}(3,2), \operatorname{ARMA}(4,3)$; bajo tres diferentes criterios de información de selección: Akaike (AIC), Hannan-Quinn (HQC) y (SBIC).

Encontramos que los modelos seleccionados bajo el criterio de Akaike para las series de migración neta masculina e inmigración neta masculina son modelos ARMA $(2,1)$; en tanto que, para las otras cuatro series el AIC indica que son caminos aleatorios; lo mismo ocurre para las seis variables migratorias con el criterio de Hannan-Quinn y con el criterio bayesiano de Schwarz: todod son caminos aleatorios (Tabla 11).

El pronóstico de la MNM para el periodo 2011-2040 a partir del modelo ARMA $(2,1)$ lleva a la Tabla 12, donde también incluimos en límite inferior y el superior del pronóstico para cada año.

Nuestro interés especial en el modelo $\operatorname{ARMA}(2,1)$ para la serie MNM radica en ser el insumo para el modelo $\operatorname{ARDL}(8,8)$, a partir del cual se puede pronosticar la MNF para los treinta años del periodo 2011-2040 (Tabla 13a y Tabla 13b). El modelo ARDL pronosticado se refleja en un MNF positivo y creciente para el periodo 2011-2020, posteriormente en el lapso 2021-2035 la cifra sigue siendo positiva pero decreciente, y en los cinco últimos años del horizonte de pronóstico la MNF es negativa (Tabla 14). 
Estacionariedad, rompimientos y tendencias de la migración internacional de México /Z.S. HERNÁNDEZ y T. SERRANO

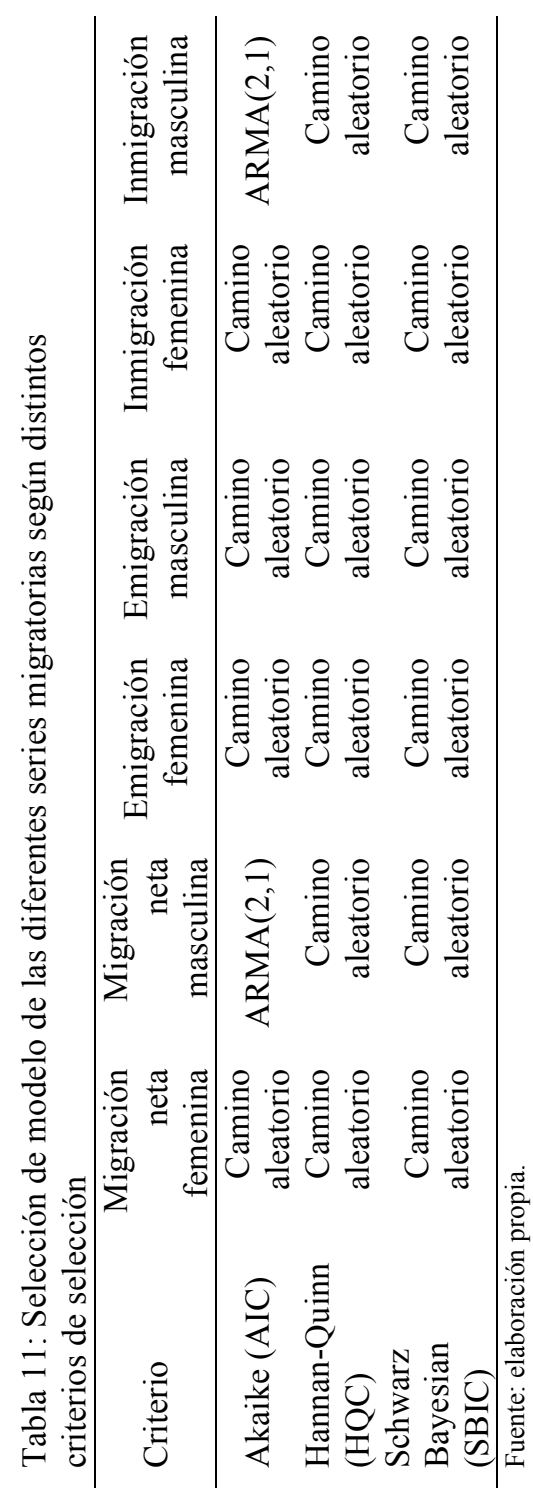


Tabla 12: Migración neta masculina de México pronosticada con modelo $\operatorname{ARMA}(2,1), 2011-2040$ (miles de personas)

\begin{tabular}{lrrr}
\hline Año & Pronóstico & Límite inferior $95 \%$ & Límite superior $95 \%$ \\
\hline 2011 & 153.586 & 89.7257 & 217.445 \\
2012 & 183.204 & 91.7648 & 274.643 \\
2013 & 210.921 & 98.1106 & 323.732 \\
2014 & 236.306 & 105.752 & 366.86 \\
2015 & 258.961 & 113.399 & 404.522 \\
2016 & 278.521 & 120.282 & 436.761 \\
2017 & 294.670 & 125.857 & 463.483 \\
2018 & 307.135 & 129.699 & 484.570 \\
2019 & 315.697 & 131.452 & 499.942 \\
2020 & 320.195 & 130.809 & 509.58 \\
2021 & 320.525 & 127.497 & 513.552 \\
2022 & 316.645 & 121.268 & 512.021 \\
2023 & 308.575 & 111.900 & 505.250 \\
2024 & 296.399 & 99.1876 & 493.610 \\
2025 & 280.262 & 82.9527 & 477.570 \\
2026 & 260.368 & 63.0461 & 457.690 \\
2027 & 236.982 & 39.3635 & 434.601 \\
2028 & 210.421 & 11.8652 & 408.977 \\
2029 & 181.053 & -19.3992 & 381.505 \\
2030 & 149.291 & -54.2643 & 352.845 \\
2031 & 115.586 & -92.429 & 323.602 \\
2032 & 80.4259 & -133.448 & 294.300 \\
2033 & 44.3203 & -176.739 & 265.380 \\
2034 & 7.7998 & -221.605 & 237.205 \\
2035 & -28.5948 & -267.266 & 210.076 \\
2036 & -64.3196 & -312.892 & 184.253 \\
2037 & -98.8358 & -357.642 & 159.970 \\
2038 & -131.618 & -400.687 & 137.450 \\
2039 & -162.162 & -441.238 & 116.914 \\
2040 & -189.992 & -478.567 & 98.5826 \\
\hline & & &
\end{tabular}

Fuente: elaboración propia. 
Tabla 13a: Migración neta femenina observada y estimada, 1968-2010, pronosticada, límites inferior y superior a partir de modelo $\operatorname{ARDL}(8,8)$, 2011-2010 (miles de personas)

\begin{tabular}{|c|c|c|c|c|}
\hline Año & MNFOBS & $\begin{array}{r}\text { MNFEST y } \\
\text { MNFPRO }\end{array}$ & $\begin{array}{r}\text { Límite } \\
\text { inferior } 95 \%\end{array}$ & $\begin{array}{r}\text { Límite } \\
\text { superior } 95 \% \\
\end{array}$ \\
\hline 1968 & 85.18 & 85.47 & 99.48 & 71.46 \\
\hline 1969 & 80.2 .0 & 78.77 & 99.02 & 58.52 \\
\hline 1970 & 67.00 & 63.24 & 88.41 & 38.06 \\
\hline 1971 & 67.02 & 57.84 & 85.66 & 30.02 \\
\hline 1972 & 57.03 & 51.10 & 78.83 & 23.38 \\
\hline 1973 & 52.04 & 47.99 & 77.13 & 18.84 \\
\hline 1974 & 50.34 & 48.28 & 81.17 & 15.39 \\
\hline 1975 & 65.04 & 79.33 & 116.19 & 42.47 \\
\hline 1976 & 61.73 & 80.52 & 121.36 & 39.67 \\
\hline 1977 & 58.38 & 80.94 & 123.58 & 38.29 \\
\hline 1978 & 55.04 & 79.02 & 122.47 & 35.56 \\
\hline 1979 & 56.63 & 63.89 & 106.98 & 20.79 \\
\hline 1980 & 51.64 & 44.83 & 88.04 & 1.63 \\
\hline 1981 & 46.66 & 32.05 & 78.03 & -13.94 \\
\hline 1982 & 46.60 & 19.45 & 69.34 & -30.45 \\
\hline 1983 & 44.90 & 15.64 & 68.40 & -37.11 \\
\hline 1984 & 39.92 & 17.37 & 70.85 & -36.11 \\
\hline 1985 & 23.43 & 0.34 & 53.16 & -52.47 \\
\hline 1986 & 11.87 & -7.13 & 45.12 & -59.37 \\
\hline 1987 & -2.97 & -13.43 & 39.16 & -66.03 \\
\hline 1988 & -14.53 & -24.05 & 29.94 & -78.04 \\
\hline 1989 & -35.94 & -29.08 & 28.53 & -86.68 \\
\hline 1990 & -75.43 & -40.19 & 21.59 & -101.97 \\
\hline 1991 & -86.99 & -43.12 & 21.92 & -108.16 \\
\hline 1992 & -96.90 & -47.98 & 18.75 & -114.70 \\
\hline 1993 & -105.17 & -57.28 & 8.67 & -123.22 \\
\hline 1994 & -110.16 & -73.63 & -9.03 & -138.23 \\
\hline 1995 & -100.36 & -107.18 & -41.31 & -173.04 \\
\hline 1996 & -110.27 & -138.27 & -65.41 & -211.13 \\
\hline 1997 & -118.55 & -167.18 & -84.12 & -250.24 \\
\hline 1998 & -125.17 & -182.53 & -92.24 & -272.82 \\
\hline 1999 & -136.73 & -187.38 & -96.17 & -278.58 \\
\hline 2000 & -240.29 & -263.75 & -175.58 & -351.93 \\
\hline 2001 & -143.41 & -155.39 & -70.04 & -240.73 \\
\hline 2002 & -112.26 & -116.75 & -27.79 & -205.70 \\
\hline 2003 & -132.03 & -122.58 & -23.88 & -221.27 \\
\hline
\end{tabular}

Fuente: elaboración propia a partir de modelo ARDL. 
Tabla 13b: Migración neta femenina observada y estimada, 1968-2010, pronosticada, límites inferior y superior a partir de modelo $\operatorname{ARDL}(8,8)$, 2011-2010 (miles de personas)

\begin{tabular}{|c|c|c|c|c|}
\hline Año & MNFOBS & $\begin{array}{r}\text { MNFEST y } \\
\text { MNFPRO } \\
\end{array}$ & $\begin{array}{r}\text { Límite } \\
\text { inferior } 95 \%\end{array}$ & $\begin{array}{r}\text { Límite } \\
\text { superior } 95 \%\end{array}$ \\
\hline 2004 & -125.52 & -113.69 & -0.54 & -226.84 \\
\hline 2005 & -50.00 & -27.88 & 98.38 & -154.14 \\
\hline 2006 & -2.41 & 38.12 & 170.02 & -93.78 \\
\hline 2007 & 25.46 & 78.47 & 205.94 & -49.00 \\
\hline 2008 & 45.12 & 95.74 & 213.22 & -21.73 \\
\hline 2009 & 68.06 & 104.85 & 219.13 & -9.44 \\
\hline 2010 & 66.36 & 78.99 & 205.13 & -47.15 \\
\hline 2011 & & 81.81 & 240.03 & -76.41 \\
\hline 2012 & & 62.59 & 247.81 & -122.64 \\
\hline 2013 & & 68.36 & 268.17 & -131.46 \\
\hline 2014 & & 112.92 & 308.96 & -83.11 \\
\hline 2015 & & 156.51 & 335.72 & -22.69 \\
\hline 2016 & & 207.68 & 378.62 & 36.74 \\
\hline 2017 & & 274.58 & 463.01 & 86.15 \\
\hline 2018 & & 313.85 & 538.55 & 89.15 \\
\hline 2019 & & 324.80 & 584.68 & 64.92 \\
\hline 2020 & & 333.05 & 607.25 & 58.85 \\
\hline 2021 & & 324.01 & 588.62 & 59.41 \\
\hline 2022 & & 293.77 & 536.29 & 51.25 \\
\hline 2023 & & 268.01 & 494.62 & 41.39 \\
\hline 2024 & & 238.73 & 474.04 & 3.42 \\
\hline 2025 & & 190.91 & 456.12 & -74.30 \\
\hline 2026 & & 145.19 & 447.92 & -157.54 \\
\hline 2027 & & 108.98 & 443.10 & -225.14 \\
\hline 2028 & & 74.14 & 416.28 & -268.00 \\
\hline 2029 & & 56.78 & 379.65 & -266.10 \\
\hline 2030 & & 65.72 & 357.22 & -225.79 \\
\hline 2031 & & 81.70 & 366.62 & -203.22 \\
\hline 2032 & & 97.28 & 422.69 & -228.12 \\
\hline 2033 & & 111.72 & 495.21 & -271.78 \\
\hline 2034 & & 107.07 & 533.18 & -319.04 \\
\hline 2035 & & 77.56 & 516.67 & -361.55 \\
\hline 2036 & & 35.70 & 457.85 & -386.45 \\
\hline 2037 & & -17.39 & 372.23 & -407.00 \\
\hline 2038 & & -81.28 & 289.14 & -451.70 \\
\hline 2039 & & -142.48 & 250.12 & -535.09 \\
\hline 2040 & & -196.90 & 255.62 & -649.43 \\
\hline
\end{tabular}

Fuente: elaboración propia a partir de modelo ARDL. 
Tabla 14: Regresión de la serie migración neta total contra repatriaciones y contra ella misma

Variable dependiente: MIGNETATOT

Método: Mínimos cuadrados

Muestra (ajustada): 19962010

Observaciones incluidas: 15 después de los ajustes

\begin{tabular}{lrrrr}
\hline Variable & Coeficiente & Error estándar & $t$-calculado & Prob. \\
\hline REPATRIAC & 0.518176 & 0.228301 & 2.269705 & 0.0443 \\
REPATRIAC(1) & -0.496906 & 0.220110 & -2.257535 & 0.0453 \\
MIGNETATOT(-1) & 0.900798 & 0.116274 & 7.747186 & 0.0000 \\
DUM & -441016.0 & 116302.1 & -3.791987 & 0.0030 \\
\hline R-cuadrado & 0.906357 & Media var. dependiente & -172508.0 \\
R-cuadrado ajustado & 0.880818 & D. E. var. dependiente & 226011.7 \\
E. E. de la regresión & 78025.47 & Criterio información Akaike & 25.59064 \\
Suma residuos cuadrado & $6.70 \mathrm{E}+10$ & Criterio inf Schwarz & 25.77945 \\
Log de verosimilitud & -187.9298 & Criterio Inf Hannan-Quinn. & 25.58863 \\
Durbin-Watson & 1.148434 & & & \\
\hline
\end{tabular}

Fuente: elaboración propia a partir de INM.

Relación entre migración neta total-repatriados, y emigración total-repatriados

El Instituto Nacional de Migración tiene estadísticas de los repatriados ordenadamente por Estados Unidos desde el año 1995 hasta 2015, sus cifras representan devoluciones, no personas; esto es, una persona pudo ser capturada varias veces al intentar ingresar a Estados Unidos. Los dos siguientes pronósticos tienen sustento en la alta correlación entre flujos de migración neta total y emigración total con repatriaciones.

El periodo de estudio es apenas de 21 años, hemos establecido previamente que las series migración neta total (MIGNETATOT) es estacionaria con un rompimiento en 1999, lo mismo para la serie emigración por sexo, aun cuando aquí utilizamos la emigración total (suma de la femenina y masculina); por su parte, al aplicar una prueba de raíz unitaria DickeyFuller aumentada a la serie repatriaciones (REPATRIAC) la conclusión es estacionariedad, por lo menos al 95 por ciento de confianza: t-calculado es -4.08 y valor crítico de MacKinnon es -3.67 al cinco por ciento de significancia.

Primero consideremos la migración neta total (MIGNETATOT) como dependiente de las repatriaciones (REPATRIAC), de las repatriaciones adelantadas un periodo (REPATRIAC(1)) — este adelanto se debe a que la personas saben o escuchan sobre lo difícil o fácil que es ingresar a Estados 
Unidos sin ser detectados por la Patrulla Fronteriza, y sobre cuestiones de inseguridad, a partir de ello deciden si deben intentar cruzar o bien por dónde es más probable no ser detectado o es más seguro-, de la migración neta total rezagada un periodo (MIGNRTATOT(-1)) y de una variable dummy (DUM) de impulso en el año 2000 (el cual está en el intervalo definido previamente para la fecha de rompimiento). Como podemos ver el ajuste es muy aceptable (Tabla 14 y Figura 7).

Esta regresión supera todas las pruebas de autocorrelación de los residuos, de los residuos al cuadrado del multiplicador de Lagrande de Breusch-Godfrey, por lo menos a cinco por ciento de significancia; lo mismo con normalidad y con heteroscedasticidad.

El pronóstico dinámico para el periodo 2011-2015 (perderemos una observación por el adelanto de la serie de repatriaciones) nos lleva a cifras con un amplio intervalo de confianza (Figura 8 y Tabla 15).

Por otra parte, el pronóstico estático de la migración neta total para el año 2011 tiene un valor de 196637 personas, con un límite superior de 371 mil personas y uno inferior de 22 mil personas.

Asimismo, estimamos la relación entre emigración total (EMITOT) y repatriaciones (REPATRIAC), repatriaciones adelantadas un periodo (REPATRIAC(1) y emigración total con un rezago (EMITOT(-1)), además de la variable dummy (DUM) del año 2000 como fecha de rompimiento. Tal estimación es aceptable estadísticamente (Figura 9 y Tabla 16).

El pronóstico de la emigración total para los años 2011-2014 indica un descenso en este indicador (Figura 10 y Tabla 17).

\section{Histórica-demográfica}

La disminución de los flujos migratorios está asociada a la reciente crisis económica acontecida en el vecino país del norte, y en los años posteriores por el mal desempeño de aquellos sectores en los que los trabajadores mexicanos se concentran más intensamente (por ejemplo, construcción y manufactura). De hecho, es el caso que durante la crisis más reciente de las condiciones del mercado de trabajo que enfrentan los inmigrantes mexicanos en Estados Unidos parecen haber empeorado aún más que para el resto de la fuerza laboral, lo que sugiere que la crisis golpeó especialmente a los sectores que tienden a emplear relativamente más mexicanos. 
Figura 7: Serie observada y ajustada de la migración neta total y residuos, 1995-2010

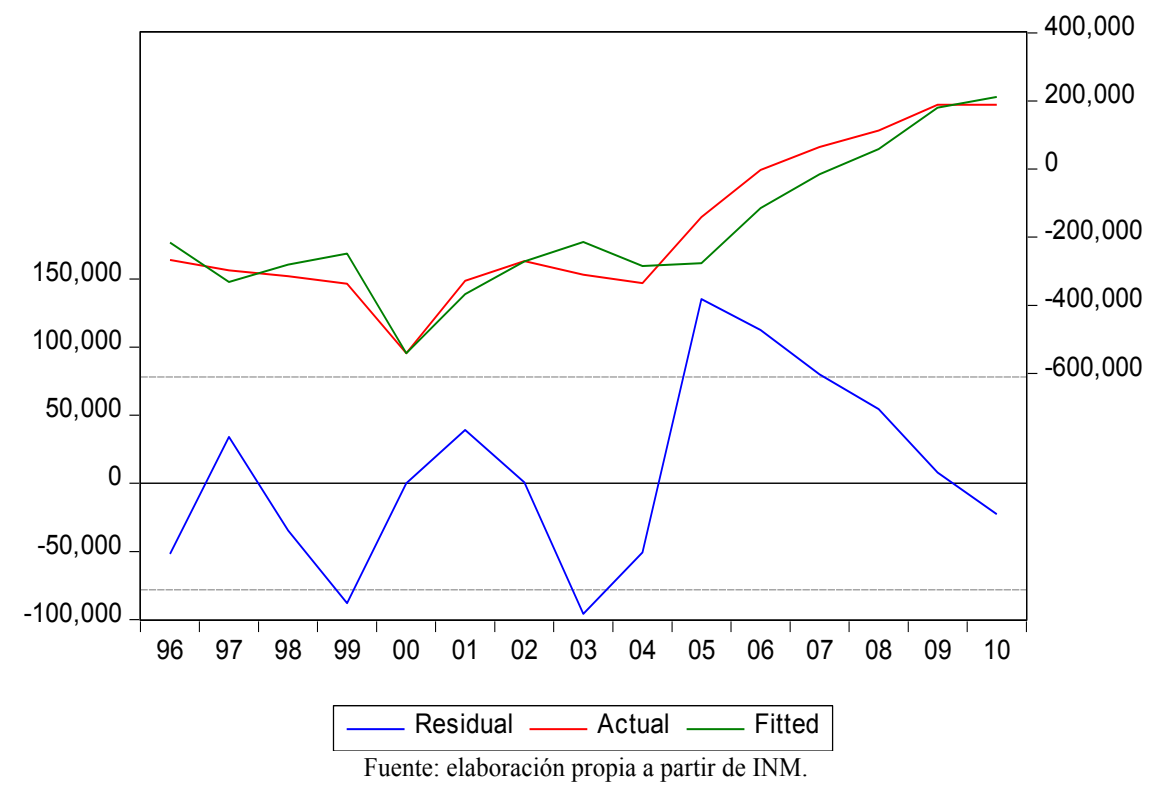

Figura 8: Pronóstico e intervalos de la serie migración neta total, 2011-2014

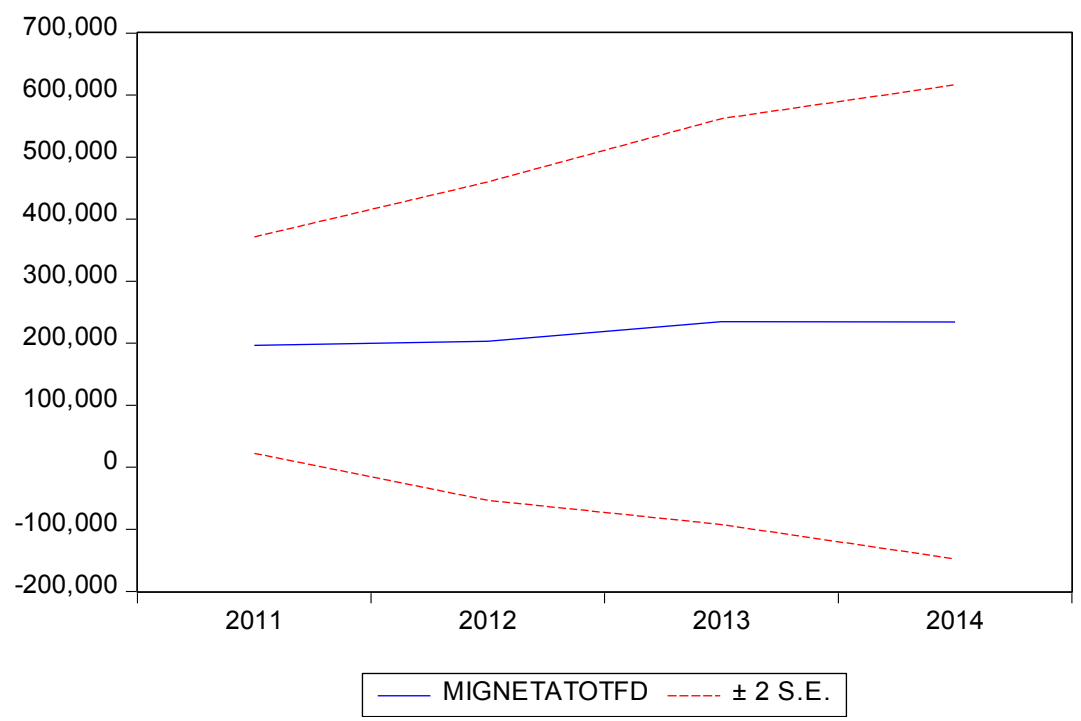

Fuente: elaboración propia. 
Tabla 15: Pronósticos y error estándar de la serie migración neta total, 2011-2014

\begin{tabular}{lrr}
\hline Año & Pronóstico & Error estándar \\
\hline 2011 & 196637 & 87241 \\
2012 & 203190 & 128259 \\
2013 & 234670 & 163564 \\
2014 & 234351 & 191096 \\
\hline
\end{tabular}

Fuente: elaboración propia.

Figura 9: Serie observada y ajustada de la emigración total y residuos, 1995-2010

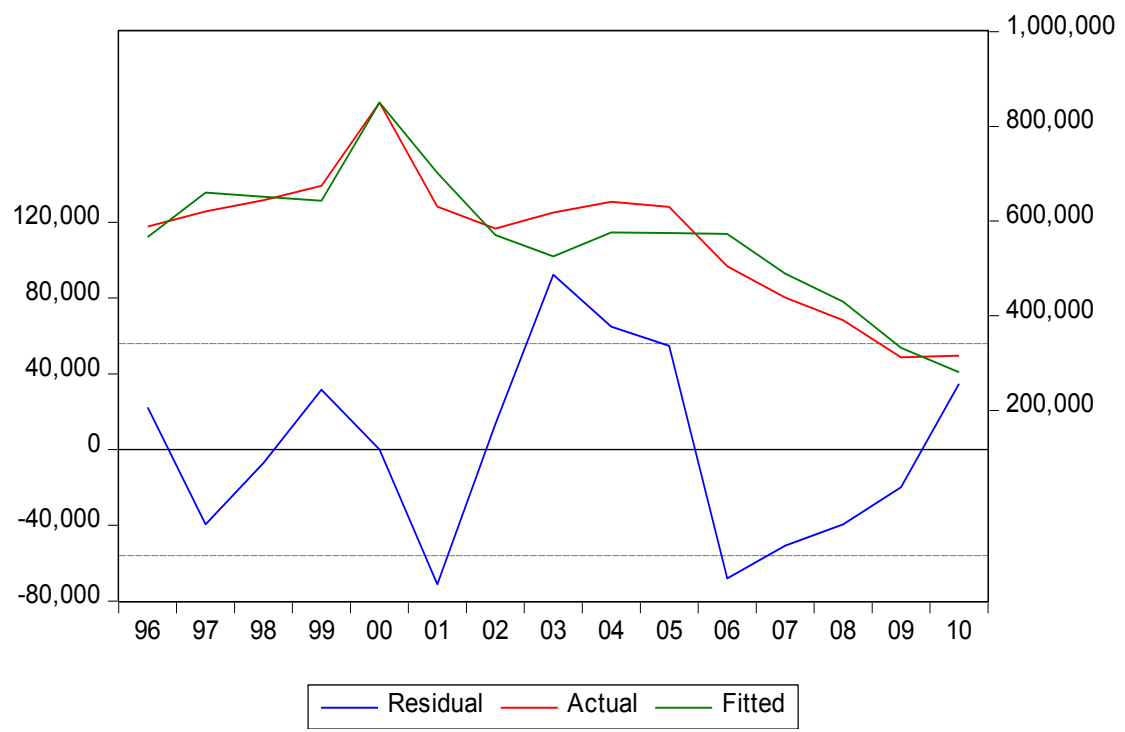

Fuente: elaboración propia

En particular, mientras que la tasa general de desempleo en Estados Unidos y la tasa de desempleo de los inmigrantes mexicanos se encontraban en niveles similares de alrededor de 4.5 por ciento durante 2006 y el primer semestre de 2007, por 2009-2010 la tasa de desempleo que enfrentan los inmigrantes mexicanos se elevó a un promedio de alrededor de 11.4 por ciento, mientras que la tasa general de desempleo alcanzó un 9.4 por ciento promedio (OCDE, 2013). 
Tabla 16: Regresión de la serie emigración total contra repatriaciones, dummy y contra ella misma

Variable dependiente: EMITOT

Método Mínimos Cuadrados

Muestra ajustada: 19962010

Observaciones incluidas: 15 después del ajuste

\begin{tabular}{lrrrr}
\hline Variable & Coeficiente & Error estándar & $t$-calculado & Prob. \\
\hline REPATRIAC & -0.284463 & 0.203706 & -1.396439 & 0.1901 \\
REPATRIAC(1) & 0.385898 & 0.166511 & 2.317560 & 0.0408 \\
EMITOT(-1) & 0.825429 & 0.093453 & 8.832547 & 0.0000 \\
DUM & 315478.7 & 92368.71 & 3.415428 & 0.0058 \\
\hline R-cuadrado & 0.883936 & Media var. dependiente & 562827.3 \\
R-cuadrado ajustado & 0.852283 & D. E. var. dependiente & 145570.9 \\
E. E. de la regresión & 55948.72 & Criterio información Akaike & 24.92544 \\
Suma residuos cuadrado & $3.44 \mathrm{E}+10$ & Criterio inf Schwarz & 25.11425 \\
Log de verosimilitud & -182.9408 & Criterio Inf Hannan-Quinn. & 24.92343 \\
Durbin-Watson & 1.322022 & & \\
\hline Fur & & & \\
\end{tabular}

Fuente: elaboración propia.

Figura 10: Pronóstico e intervalos de la serie emigración total, 1995-2014

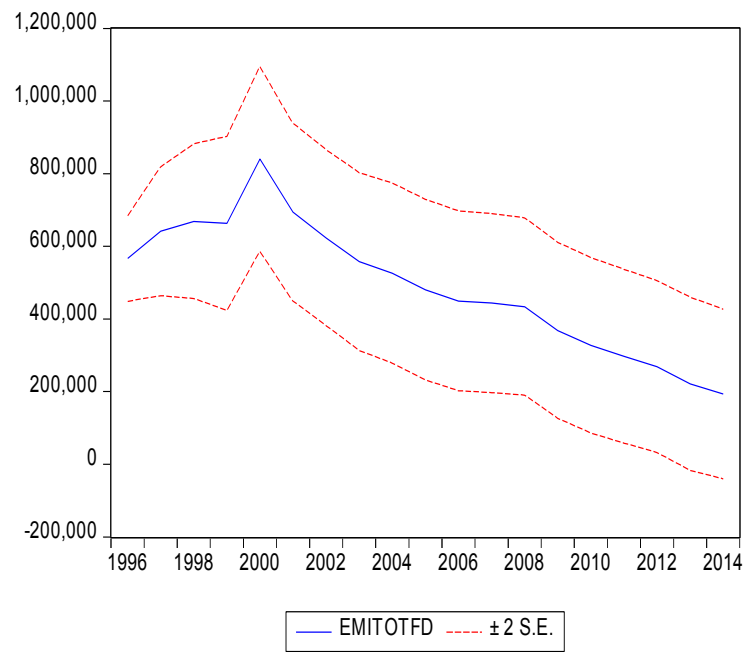

Forecast: EMITOTFD

Actual: EMITOT

Forecast sample: 19952015

Adjusted sample: 19962014

Included observations: 19

Root Mean Squared Error $\quad 60365.92$

Mean Absolute Error

45763.84

Mean Abs. Percent Error $\quad 8.441769$

Theil Inequality Coefficient $\quad 0.052570$

Bias Proportion $\quad 0.031315$

Variance Proportion $\quad 0.012242$

Covariance Proportion $\quad 0.956443$

Theil U2 Coefficient $\quad 0.763803$

Symmetric MAPE

8.658202

Fuente: elaboración propia. 
Tabla 17: Pronóstico y error estándar de emigración total femenino, 2011-2015

\begin{tabular}{lrr}
\hline Año & EMITOTFD & Error Estándar \\
\hline 2011 & 57997.72 & 536112.67 \\
2012 & 32178.39 & 504907.71 \\
2013 & -17392.40 & 459040.85 \\
2014 & -40588.44 & 426846.20 \\
\hline
\end{tabular}

Fuente: elaboración propia.

Esta situación es resultado, principalmente, debido a que la recuperación del empleo en Estados Unidos aún carece de impulso, no se han recuperado todos los puestos de trabajo que se perdieron en la recesión, de los 7.7 millones de puestos de trabajo que se perdieron durante la crisis, solo se recuperaron 6.4 millones hasta 2013. A finales de este año, todos los principales indicadores del mercado laboral, tales como tasas de empleo y desempleo, estaban aún por debajo de sus niveles previos a la recesión. Pero, los indicadores de las personas hispanas no nacidas en Estados Unidos no han levantado desde 2011, el empleo de los inmigrantes hispanos ha estado en un punto muerto. En el sector de la construcción, los hispanos perdieron 686 mil puestos de trabajo durante la recesión y recuperaron sólo 74 mil de ellos de 2009 a 2013. La gran mayoría de los puestos de trabajo adquiridos de 2009 a 2013 ascendió de 2.3 a 2.8 millones ocupados por los hispanos nacidos en Estados Unidos. Mientras tanto, los cerca de 450 mil nuevos puestos de trabajo adquiridos por los inmigrantes hispanos se remontan a los primeros dos años de la recuperación, a partir de 2009 a 2011. Es decir, existe una sensible disminución de la demanda de mano de obra migrante. Es por ello, que bajo este contexto, no hay razones para pensar que por el momento haya un incremento de la migración de mexicanos hacia ese país, la migración está en periodo de impase que se caracteriza por un descenso de la migración de retorno y una estabilización del flujo de migrantes a Estados Unidos partir de 2010. Por último, los últimos acontecimientos políticos pueden reforzar aún más el descenso de migrantes y una caída más pronunciada de la migración de retorno, pues con la administración de Trump pueda que las condiciones para retornar se vuelvan peores.

A lo anterior, se debe agregar que los hijos de inmigrantes mexicanos ocupan posiciones que antes eran para los indocumentados; es decir, hay sustitutos de estos migrantes ya en Estados Unidos México también ha tenido un avance en cuestiones educativas durante los últimos años, las po- 
líticas de contención en la frontera, los problemas de inseguridad, las dificultades económicas, los altos costos para cruzar relativamente seguros; así como la profundización de las políticas más severas en cuestiones migratorias apuntan por lo menos a una estabilización de los flujos migratorios.

\section{Conclusiones}

La incorporación de rompimientos en las series migratorias lleva a concluir que son estacionarias, tales cambios reflejados en los parámetros estuvieron basados en factores como el boom demográfico de los sesenta y la crisis de los ochenta; el segundo rompimiento es previo a los sucesos terroristas en Estados Unidos y puede estar relacionado con políticas migratorias más severas contra personas indocumentadas. Lo relevante del último rompimiento, con el cual cambia la tendencia de todas las series hacia una donde cada vez hay menos migrantes netos desde México, es investigar si tiene asociación con el aumento en la inseguridad en la frontera.

El apartado de pronósticos son simples ejercicios basados en demasiados supuestos: en el lineal se asume que no habrá otro rompimiento durante el periodo de pronóstico, en el dinámico es supuesta una relación basada sólo en el pasado de las series consideradas y en el mantenimiento de la relación entre ellas, en el pronóstico basado en las repatriaciones tenemos una relación sustentada en reportes donde se establece una caída desde el máximo histórico en 1999, mientras en 2015 se registra el mínimo histórico. En todos los casos los intervalos de confianza son muy amplios.

Cabe preguntarse los efectos de las políticas migratorias a imponer por la administración federal de Estados Unidos y por las administraciones estatales a inicios de 2017, los cuales pueden afectar aun más estos flujos. Será la información censal nuevamente la cual despejará nuestras incógnitas y abrirá otras nuevas con respecto a la migración internacional.

\section{REFERENCIAS BIBLIOGRÁFICAS}

Banco Mundial., s.f., Indicadores, Banco Mundial, consultado el 15/01/2016, recuperado de http://datos.bancomundial.org/indicador

Bustamante, J., 1986, El marco teórico de la circularidad migratoria, su validación empírica. Fondo de Cultura Económica.

Calavitia, K., 1989, "El debate de la política estadounidense de migración: análisis crítico y opciones para el futuro", en J. Bustamante and W. Cornelius, Flujos migratorios de mexicanos hacia Estados Unidos. Fondo de Cultura Económica. 
Carrion-i-Silvestre, J. L., Del Barrio-Casto, T. and López-Bazo, E., 2005, "Breaking the panels. an application to the GDP per capita", in Econometrics Review, 159-175.

Dickey, D. and Fuller, W., 1979, "Distribution of the Estimators for Autoregressive Time Series with a Unit Root", in Journal of the American Statistical Association, 34, 427-431.

Figueroa-Hernández, E. and Pérez-Soto, F., 2011, "El proceso de asentamiento de la migración México-Estados Unidos", en Papeles de Población, 17(68), 161-190.

García-Guerrero, V. M., 2016, A probabilistic method to forecast the international migration of Mexico by age and sex, en Papeles de población, 22(88), 113-140.

Giles, D., 2013, “Econometric's beat" Dave Giles Blog. Consultado el 6/03/2013, Recuperado el 2015, de ARDL models: part I: http://davegiles.blogspot. $\mathrm{mx} / 2013 / 03 /$ ardl-models-part-i.html

Hansen, B. E., 2001, "The New econometrics of Structural Change: Dating Breaks in U. S. Labor Productivity", in Journal of economic Perspectives, 15(4), 117-128.

INEGI, 2016, Información de la Migración Internacional con Datos de la ENOE al tercer trimestre de 2015, consultado el 28/01/2016, recuperado de http://www. inegi.org.mx/saladeprensa/boletines/2016/especiales/especiales2016_01_10.pdf

Johnston, J. and Dinardo, J., 2001, "Modelos autorregresivos y con retardos distribuidos", en Métodos de econometría, Vicens-Vives, págs. 282-329.

López, G., 1986, La casa dividida. Fondo de Cultura Económica.

Massey, D. S., Pren, K. A. and Durand, J., 2009, "Nuevos escenarios de la migración México-Estados Unidos: Las consecuencias de la guerra antiinmigrante", en Papeles de Población, 15(61), 101-128.

Pesaran, Shin, Y. and Smith, O., 2001, "Bounds Testing Approaches to the Analysis of Level Relationships", in Journal of applied econometrics.

Pyndick, J. and Rubinfeld, P., 2001, Econometría: modelos y pronósticos, McGraw Hill, Madrid.

Shin, Y., Pesaran, M. H. And Smith, R. J., 2001, "Bounds Testing Approaches to the Analysis of Level Relationships", in Journal of Applied Econometrics, 16, 289-326. 


\section{RESUMEN CURRICULAR DE LOS AUTORES}

Zeus Salvador Hernández Veleros

Profesor investigador de la Universidad Autónoma del Estado de Hidalgo. Doctor en Economía por la Universidad de Barcelona. Título de tesis "Crecimiento Económico y Fluctuaciones Cíclicas: La Importancia de la Estabilidad Macroeconómica". Subdirector de Métodos Cuantitativos en el Instituto Nacional de Migración (1996-1997). Publicaciones recientes: "Modelos de crecimiento, estacionariedad y rompimientos: comparación entre las tendencias de crecimiento de las economías de la OCDE y las de los países menos desarrollados." El Trimestre Económico, vol. LXXXIII (4), núm. 332, octubre-diciembre de 2016, pp. 635-678.Artículo: "El puerto del cuarto polo de desarrollo: relación de largo plazo entre la API de Lázaro Cárdenas y el PIB de México, 1995-2013." Paradigma Económico, 7,1, 2015.

Dirección electrónica: zshveleros@yahoo.com

\section{Tomás Serrano Avilés}

Profesor investigador de la Universidad Autónoma del Estado de Hidalgo. Doctor en Ciencias Sociales por el Colegio de la Frontera Norte, Profesor investigador de la Universidad Autónoma del Estado de Hidalgo, cultiva la línea migración internacional y ambiente. Coordinador del Área Académica de Sociología y Demografía de la UAEH.

Dirección electrónica: tomascongreso@hotmail.com 